\title{
Nanoantenna Enhanced Terahertz Interaction of Biomolecules
}

\author{
Subham Adak ${ }^{1}$ and Laxmi Narayan Tripathi ${ }^{1, *}$ \\ ${ }^{1}$ Department of Physics, Birla Institute of Technology, Mesra, Ranchi-835215, Jharkhand, India.
}

(Dated: March 11, 2019)

\begin{abstract}
Terahertz time-domain spectroscopy (THz-TDS) is a non-invasive, non-contact and label-free technique for biological and chemical sensing as THz-spectra is less energetic and lies in the characteristic vibration frequency regime of proteins and DNA molecules. However, THz-TDS is less sensitive for detection of micro-organisms of size equal to or less than $\lambda / 100$ (where, $\lambda$ is wavelength of incident $\mathrm{THz}$ wave) and, molecules in extremely low concentrated solutions (like, a few femtomolar). After successful high-throughput fabrication of nanostructures, nanoantennas and metamaterials were found to be indispensable in enhancing the sensitivity of conventional THz-TDS. These nanostructures lead to strong $\mathrm{THz}$ field enhancement which when in resonance with absorption spectrum of absorptive molecules, causing significant changes in the magnitude of the transmission spectrum, therefore, enhancing the sensitivity and allowing detection of molecules and biomaterials in extremely low concentrated solutions. Hereby, we review the recent developments in ultra-sensitive and selective nanogap biosensors. We have also provided an in-depth review of various high-throughput nanofabrication techniques. We also discussed the physics behind the field enhancements in sub-skin depth as well as sub-nanometer sized nanogaps. We introduce finite-difference time-domain (FDTD) and molecular dynamics (MD) simulations tools to study $\mathrm{THz}$ biomolecular interactions. Finally, we provide a comprehensive account of nanoantenna enhanced sensing of viruses (like, H1N1) and biomolecules such as artificial sweeteners which are addictive and carcinogenic.
\end{abstract}

\section{CONTENTS}

I. Introduction

II. High-Throughput Wafer-scale Fabrication Techniques

III. Terahertz-Field Enhancement using Nanoantennas

A. THz Field Enhancement in Metal Nanogaps at Sub Skin-Depth Regime

B. THz Field Enhancement in Sub-Nanometer and Angstrom Metallic gaps: Quantum Regime

C. The Finite-Difference Time-Domain (FDTD) Algorithm : A Computational

Electromagnetism Approach

IV. THz-TDS and Sensing of Molecules and Biomolecules

A. Computational THz-TDS using Molecular Dynamics (MD) Simulation

B. THz-Nanoantenna and Metamaterials Sensing of Micro-organisms, Organic- and Biomolecules

V. Conclusion and Future Outlook

VI. Acknowledgement

VII. Conflict of Interest

VIII. Keywords

References

\section{INTRODUCTION}

Optical antennas, ${ }^{1,2}$ devices converting free optical radiations into localized ones, and vice versa are important components in modern nanophotonic devices. In the recent decade, metallic nanoantenna has attracted much consideration with the significant advancement of nanofabrication technologies, which provide the feasibility of fabrication at nanometre resolution, enabling fascinating nano-optoelectronic devices. Since metals exhibit finite conductivities and plasmonic effect at optical frequencies, therefore the optical field response is dictated by the resonance oscillation of electron clouds (surface plasmons, or SP) in the metal, coupling with the incident photon and sustaining surface plasmon polaritons (SSPs). ${ }^{3,4}$ Henceforth, at optical frequencies a nanoantenna, depending upon the material properties, resonates for considerably shorter effective wavelength, rather than half-wavelength of free-space. 5,6

Electromagnetic interaction of nanoantennas has lead to huge local field enhancement and confinement in nano-size volumes, enabling broadband application in nano-optics. ${ }^{7-15}$ Terahertz (THz) wave funnelling through nanogaps/nanoantennas has resulted huge field enhancement. ${ }^{16-40}$ Figure 1 summarizes the various investigations performed to study the $\mathrm{THz}$ field enhancement with different gap sizes. Such investigations have lead to the development of the terahertz technology, which are now exploited in electronics, ${ }^{41-46}$ photonics, ${ }^{47-49}$ medical sciences, ${ }^{50-52}$ military, ${ }^{53-55}$ security $^{56,57}$ and conservation of cultural heritages. ${ }^{58,59}$ These studies also lead to the improvisation of terahertz devices. ${ }^{60-76}$

Terahertz time-domain spectroscopy (THz-TDS) ${ }^{77-82}$ 


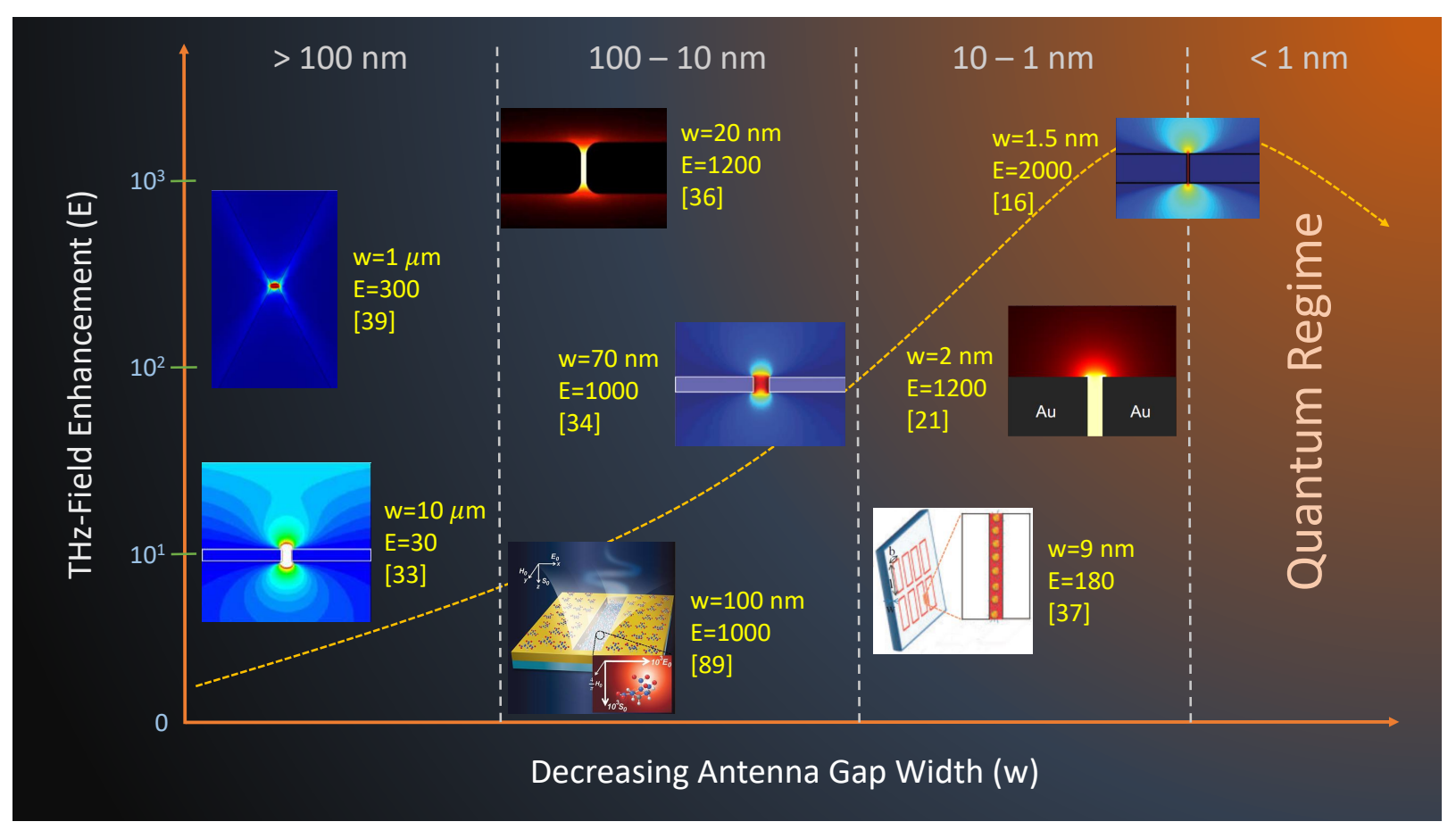

Figure 1. THz-field enhancement profile with decreasing gap width.

is a non-invasive and one of the most widely used technique in biosensing $80,83-87$ because the THz-spectra are less energetic (about a few milli-electronvolts) lies in the characteristic vibration frequency regime of proteins and DNA molecules. Terahertz nanoantennas provide us label-free and ultra-sensitive sensing of molecules $^{88,89}$ and biomolecules ${ }^{37,81,90-94}$ as the nanoantennas focus the $\mathrm{THz}$ waves into an extremely confined volume where we can place our analytes at very low concentrations. Significant changes in the magnitude of the transmission spectrum were observed in the absorptive molecules when the resonance mode of the waveguide matches its absorption spectrum, 36,83 enhancing the sensitivity of THz-resonators based sensing devices, therefore making it feasible for detection of biomolecules at extremely low concentration.

In this review work, we describe the high-throughput fabrication techniques of $\mathrm{THz}$ nanoantenna via. photolithography, atomic layer lithography, pattern and peel method, self-assembly lithography etc. Then we discuss the $\mathrm{THz}$ field enhancement using metal nanogap antenna where we discuss the field enhancement beyond the skin depth of the metal up to the quantum regime. Then we briefly discuss finite-difference timedomain (FDTD) methods for calculation of field enhancement across the nanogap. At the last, we describe the THz-TDS for sensing the biomolecules due to the field enhancement across the nanogap. We also showed how a molecular dynamics (MD) simulation can be a versatile tool to estimate the terahertz absorption and vibrational density of states (VDOS). Then we

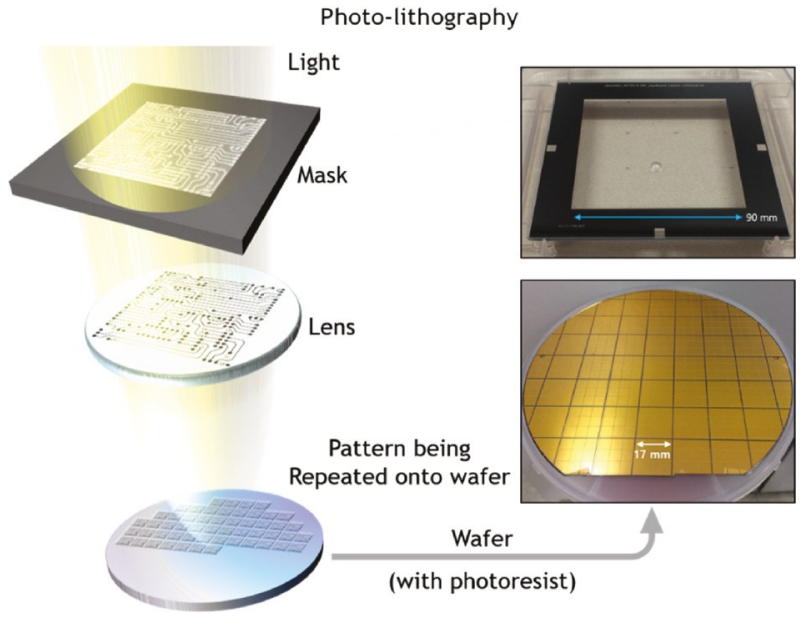

Figure 2. Photolithography : A high resolution and high throughput technique for nanogap structures. Reprinted with permission from Ref. ${ }^{23}$. (C) 2018, De Gruyter.

have taken real examples from various literature that how $\mathrm{THz}$ nanoantenna and metamaterials can be used to enhance the terahertz biomolecular sensing.

\section{HIGH-THROUGHPUT WAFER-SCALE FABRICATION TECHNIQUES}

A nanogap must have a reliable THz-field interaction and plasmonic characteristics (like, field enhancement 

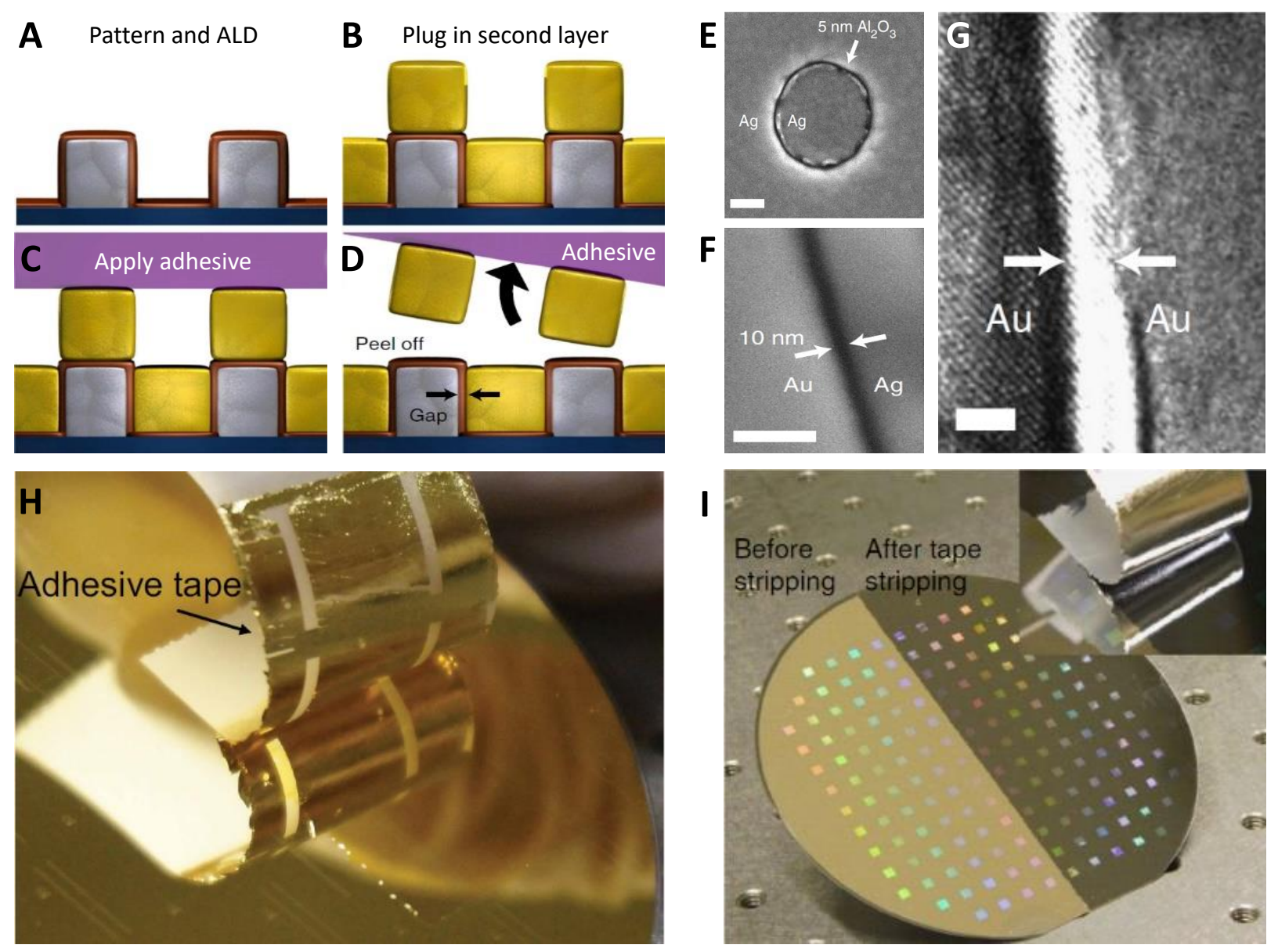

Figure 3. Atomic layer lithography. (A) Thin $\mathrm{Al}_{2} \mathrm{O}_{3}$ layer coated on a patterened substrate using ALD. (B) Metal evaporation plugging second metal layer. (C,D) Removing excess metal by using an adhesive. Scanning electron micrograph (SEM) of (E) 5 $\mathrm{nm}$ gap in $\mathrm{Ag}$-film (F) $10 \mathrm{~nm}$ gap between $\mathrm{Au}$ and $\mathrm{Ag}$, and (G) $9.9 \AA \mathrm{Au}-\mathrm{Al}_{2} \mathrm{O}_{3}$ - $\mathrm{Au}$ vertical nanogap. Reprinted with permission from Ref. ${ }^{95}$. ( 2013, Nature Communications. (H) Adhesive tape planarization of nanogap. Adapted with permission from Ref. ${ }^{21}$. (C) 2014, Springer Nature. (I) Planarization of half Si-wafer by using an adhesive. Reprinted with permission from Ref. ${ }^{95}$. (c) 2013, Nature Communications.

and non-linearity). But fabricating high throughput wafer-scale nanogaps is one of the most challenging part of the job. To achieve sufficiently intense far-field signals, nanogap arrays should be fabricated over large sample area. Several methods like: fs-laser beam machining, ${ }^{17,96-98}$ focussed ion beam, ${ }^{17,40,99-104}$ photolithography, 17,105-108 electron-beam (e-beam) lithography, ${ }^{17,109-115}$ nanosphere lithography, 17,116 sidewall lithography, ${ }_{1}^{117}$ nanoimprint, $17,118-122$ pattern and peel method ${ }^{123}$ and atomic layer lithography ${ }^{17,41,95,124-126}$ have been developed for high resolution patterning over large area of sample.

Photolithography ${ }^{17,23}$ (Fig. 2) is one of the most highthroughput methods for fabricating nanogap structures. In photolithography, a desired polymer pattern is patterned using ultraviolet (UV) light and a photomask. This polymer pattering is formed by the physical change of a photo-sensitive material, known as photoresist (PR).
Polymers added to PR decides the solubility under UV exposure. A PR which consist of an insoluble polymer which on UV exposure, changes into a soluble polymer, is called a positive PR and a PR which consist of a soluble polymer which on UV exposure, polymerizes and becomes insoluble, is called a negative PR. Therefore by using the desired PR, wafer-scale metal structures are fabricated after performing a metal deposition process on patterned resists and lift-off of the unwanted metal film.

The lithography method discussed earlier are limited to fabrication of nanometer-gaps up to a few nanometers. To study the nano- and sub-nanoscale optics, we need better lithography techniques to fabricate sub-10 nm wide gap. Many researchers have reported the significance of deposition of an atomically thin insulating layer or sacrificial layer in fabricating small gaps between two metal structures. ${ }^{127-132}$ Chen et al. $^{95}$ developed a pioneering method for fabricat- 


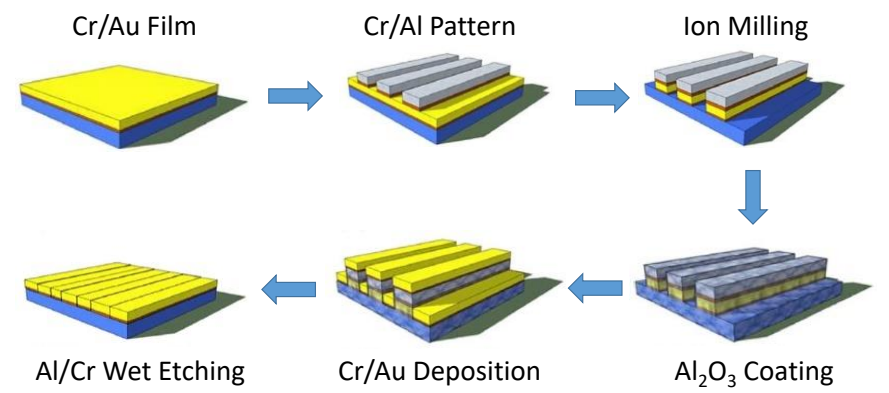

Figure 4. High-throughput fabrication technique of nanometer-scale gaps introduced by Jeyong et al. Adapted with permission from Ref. ${ }^{22}$. (C) 2014, Springer Nature.

ing vertical nanogaps known as atomic layer lithography (Fig. $3(\mathrm{~A}-\mathrm{D}))$. Figures $3(\mathrm{E}-\mathrm{G})$ are the scanning electron micrographs (SEM) of $5 \mathrm{~nm}$ gap in $\mathrm{Ag}$ film, $10 \mathrm{~nm}$ gap between $\mathrm{Au}$ and $\mathrm{Ag}$, and $9.9 \AA \mathrm{Au}$ - $\mathrm{Al}_{2} \mathrm{O}_{3}-\mathrm{Au}$ vertical nanogap, respectively, which are fabricated using atomic layer lithography technique. This method also includes a new planarization scheme which eliminates the background light transmission, enabling background-free transmission measurements. This lithography technique is the combination of atomic layer deposition (ALD) and 'plug-and-peel' adhesive tape planarization and is a three-step process. The first step includes the pre-patterning of metal using conventional lithography techniques, the second step includes the sidewall formation of materials like $\mathrm{Al}_{2} \mathrm{O}_{3}$ or $\mathrm{SiO}_{2}$ by ALD, and the third step includes the 'plug-andpeel' taping process for planarization of metal plugs. Therefore, atomic layer lithography is a high-resolution patterning method with large scale uniformity.

Jeong et al. ${ }^{22}$ improvised the atomic layer lithography technique which is demonstrated in Fig. 4. In this lithography technique, conventional photolithography and liftoff process were used to pattern $30 \mathrm{~nm} \mathrm{Cr} / 150$ $\mathrm{nm} \mathrm{Al}$ layer on the $3 \mathrm{~nm} \mathrm{Cr} / 100 \mathrm{~nm}$ gold-sapphire substrate. The $\mathrm{Cr}-\mathrm{Al}$ double layer acts as a sacrificial layer which will be etched out later, removing excess metal and making the structure planar. As compared to Al, $\mathrm{Au}$ is less-resistant to ion beam therefore, excluding the $\mathrm{Au}$ film beneath $\mathrm{Al}-\mathrm{Cr}$ layer, the exposed Au layer is removed using ion milling process. Uniform ALD of $\mathrm{Al}_{2} \mathrm{O}_{3}$ is performed over the whole structure with a thickness of nanometer accuracy. After that, the second layer of $\mathrm{Au}$ with adhesive $\mathrm{Cr}$ is deposited, filling the trenches. Finally, $\mathrm{Al} / \mathrm{Cr}$ wet etching is performed which removes the overhanging $\mathrm{Au}$ and $\mathrm{Al}$ layers, exposing the dielectric gaps. This lithography technique can be used to fabricate large-scale nanogaps with ultrahigh aspect ratio.

Tripathi et al. ${ }^{133}$ developed self-assembled lithography process by which they fabricated quantum dots (QDs) nanogap metamaterials (Fig. 5). In this lithography process, photolithography was used to pattern PR on a clean and dried sapphire substrate. With the help of a thermal evaporator (evaporation rate $=1 \AA / \mathrm{sec}$ ), a chrome layer of thickness $10 \mathrm{~nm}$ was deposited over the PR pattern. Following the process, a silver film of thickness $200 \mathrm{~nm}$ was evaporated over the chrome layer. After the evaporation of first silver layer, the whole sample was then dipped in acetone sonicated for 2-minutes at $150 \mathrm{~W}$ and $36 \mathrm{kHz}$, lifting off the PR pattern. After sonication, the sample was washed with isopropyl alcohol and dried using $\mathrm{N}_{2}$ gas. The dried sample was then dipped into a toluene solution of OT (1-8 octanedithiol) functionalized QDs, resulting in the formation of a selfassembled monolayer of QDs. The resulting sample was again dried using $\mathrm{N}_{2}$ gas and a second silver layer of same/higher thickness was deposited over the selfassembled monolayer. Then by using a scotch tape ${ }^{95}$, the second layer of silver was taped-off, leaving a vertical metal nanogap filled with a monolayer of QDs.

\section{TERAHERTZ-FIELD ENHANCEMENT USING NANOANTENNAS}

In $\mathrm{THz}$ frequency, a metal film is not a perfect conductor as well as infinitely flat. Equivalence of THz-electric field enhancement in metal nanogap ${ }^{18,28,34,35,134-136}$ and magnetic field enhancement around metallic nanoantennas ${ }^{137-140}$ can be shown by using Babinet's principle. ${ }^{141-145}$ This principle is still used implicitly in different areas of THz research. 22,27,36,38,42,93,103,107,146-168 Sommerfeld's half-plane problem ${ }^{169-171}$ is also used to study the light-aperture interaction theoretically. Bethe $^{172}$ and Bouwkamp ${ }^{173,174}$ reconsidered the Sommerfeld's halfplane problem for small apertures in infinitely thin perfect conducting plates. Further studies were performed on the light interaction with system of periodic apertures. ${ }^{175}$ Later, extensive studies were performed on enhanced light transmission through the apertures in various spectral regime. ${ }^{19,31,32,102,176-180}$ Theoretical approaches like: coupling-mode theory, ${ }^{135,181-188} \mathrm{mi}-$ croscopic models, ${ }^{28,29,189}$ transfer-matrix method ${ }^{190,191}$ and capacitor model, ${ }^{134}$ were combined with numerical methods ${ }^{76,192,193}$ to solve the enhanced light transmission and huge field enhancement around metallic apertures.

When an electromagnetic wave is incident normally on a perfectly conducting metallic plane, an induced current is developed on the surface, which reflects light back, with no charge accumulation anywhere. ${ }^{34}$ When this plane is divided into two perfectly conducting Sommerfeld half planes, macroscopic accumulation of charges take place at the edges with a length scale of one wavelength, such that the surface charge density $(\sigma)$ depends as a time function for small values of $\mathrm{x} \ll \lambda$, ${ }^{34}$ 


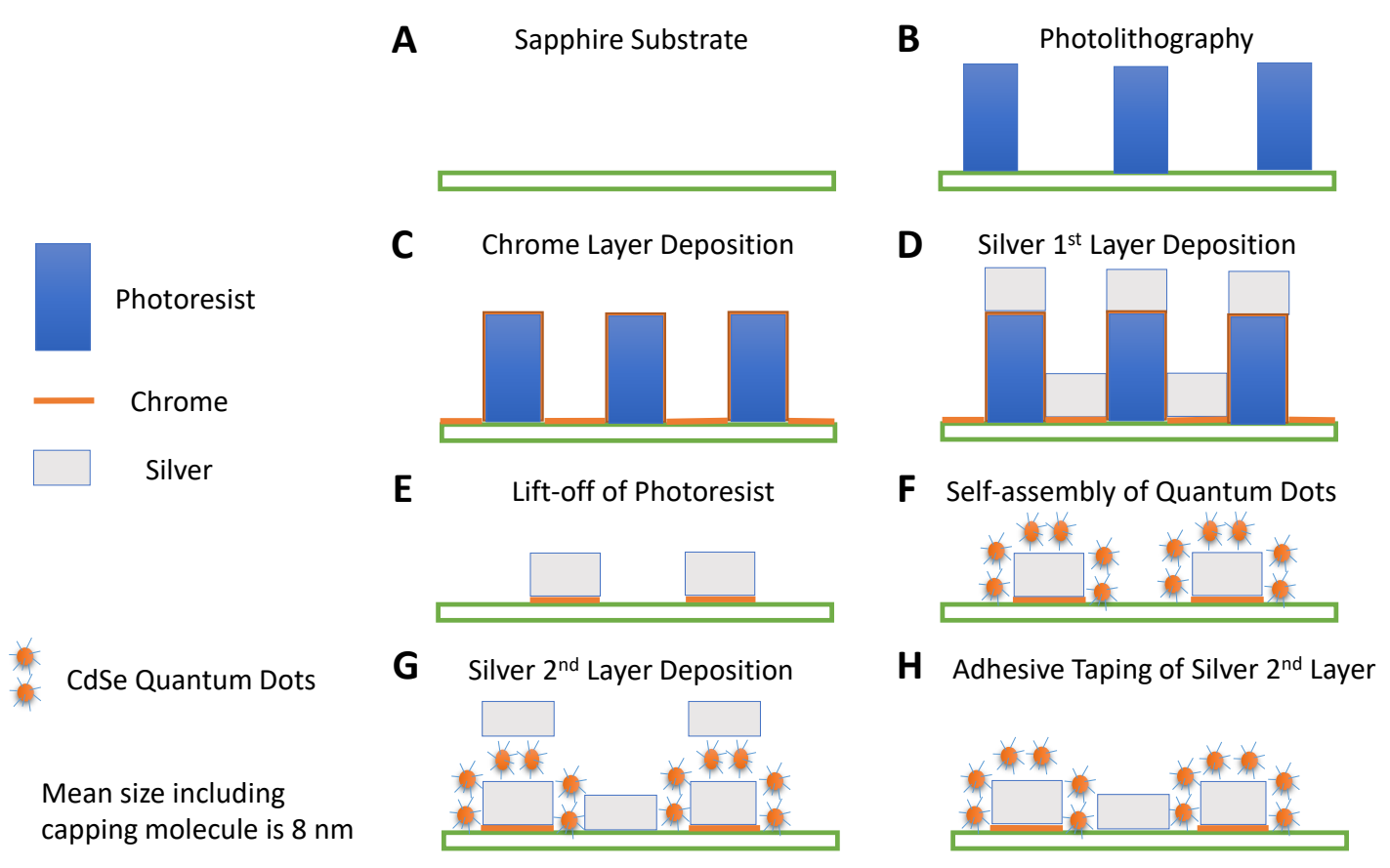

Figure 5. Self-assembly lithography technique. (A) Clean and dried sapphire substrate. (B) PR patterned using photolithography. (C) $10 \mathrm{~m}$ chrome layer deposition over PR pattern. (D) Evaporation of silver film of thickness 200 nm over the chrome layer. (E) Sonication and lift-off of PR after first silver layer deposition. (F) Self-assembly of QD monolayer over the first layer of metal. (G) Second silver layer deposition after self-assembly of QD monolayer. (H) QDs filled nanogap metamaterial after taping-off second silver layer. Adapted with permission from Ref. ${ }^{133}$. (C) 2015, Optical Society of America.

given by

$$
\sigma(x, t)=\frac{\epsilon_{0}}{\sqrt{2} \pi} \sqrt{\frac{\lambda}{x}} E_{0} e^{-i(\omega t+\pi / 4)}
$$

Here, $\epsilon_{0}, E_{0}, \omega$ and $x$ describes the permittivity of vacuum, the incident electric field, the angular frequency and distance from the edge, respectively. ${ }^{144}$ The charge singularity, at $x=0$, for this half plane is very feeble and disappears with integration. When the two metallic half planes are brought close together, an electrostatic force will be experienced by the charges due to their counter members across the gap, pulling the charges towards the edge, and developing a strong electric field across the gap. As the gap shrinks, more charges gets accumulated at the edges as the light-induced currents becomes more and more stronger. This increases the surface charge density at the edges as shown in Fig.6 B - C. This system can be portrayed as a line-capacitor driven by the light-induced alternating current as shown in Fig. 6 (A).

Numerous studies have reported the monotonic enhancement of the electric field with decreasing gap size. $^{34,93,95,145}$ Further studies have also reported the decrease in field enhancement when the gap size decreases and enters a quantum regime. ${ }^{24,194-199}$ Increase in field enhancement beyond sub-skin depth is also reported. . $^{16,23,34}$ In this section of the review, we discuss the $\mathrm{THz}$ field enhancement for gaps beyond skin-depth and also in the quantum regime. We also discuss the classical limit of field enhancement of single nanoslit before entering quantum regime, and about finitedifference time-domain algorithm, which is a computational approach developed for the quantitative estimation of far- and near-field enhancement measurements.

\section{A. THz Field Enhancement in Metal Nanogaps at Sub Skin-Depth Regime}

In this part of the section, we discuss the skindepth physics ${ }^{23}$ and the results reported in various literatures ${ }^{16,34}$. Consider two perfectly conducting metallic planes of thickness $h^{16,134}$ kept at a distance $w$ from each other, forming a metal-air-metal nanogap. An electromagnetic wave is incident normally on the metal nanogap whose electric field has a magnitude of $\mathrm{E}_{0}$. Then the ultimate field enhancement in a high-aspect ratio $(w / h \gg 1)$ is given by ${ }^{23}$

$$
E_{\text {enhancement }}=\left|\frac{E}{E_{0}}\right|=\frac{\lambda}{\pi h}
$$

where, $E$ denotes the electric field at the gap and $\lambda$ denotes the wavelength of the incident electromagnetic wave in vacuum. If a dielectric material of permittivity 
A

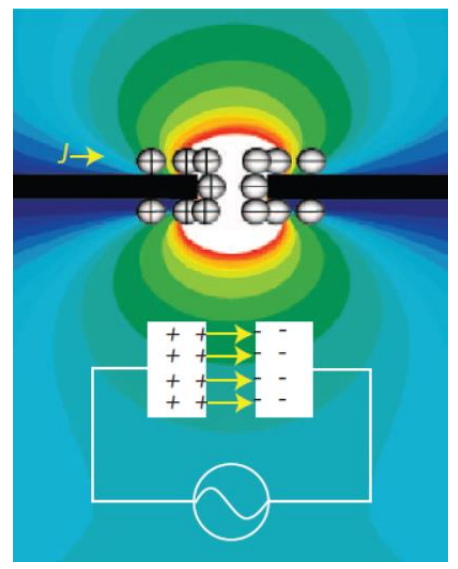

B
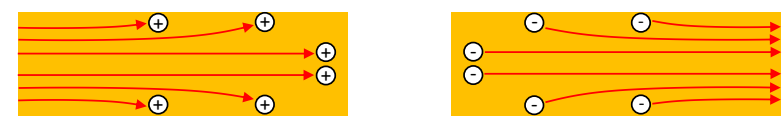

C

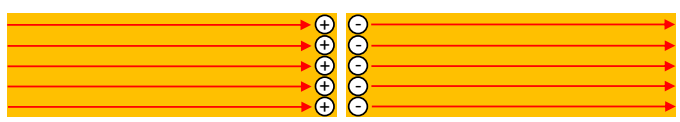

Figure 6. Capacitor-model representation of a nanogap. (A) Nanogap gets charged just like a line-capacitor due to lightinduced alternating current $J$. Hence, the electric field enhances which is shown by gradual colour contour. Reprinted with permission from Ref. ${ }^{34}$. (C) 2009, Springer Nature. Schematics of charge accumulation near metal edges and corresponding current density induced inside metal film of two gap-sizes (B) $w \sim h(\ll \lambda)$ and (C) $w \ll h(\ll \lambda)$. Adapted with permission from Ref. ${ }^{16}$. (C) 2017, American Physical Society.

$\epsilon$ is filled inside the gap, Eq. 2 modifies to

$$
E_{\text {enhancement }}=\left|\frac{E}{E_{0}}\right|=\frac{\lambda}{\pi h} \frac{\epsilon_{0}}{\epsilon}
$$

where, $\epsilon_{0}$ denotes the permittivity of vacuum. From the above equations, it can be said that the field enhancement $\left(E_{\text {enhancement }}\right)$ is independent of metal characteristics.

Considering an electromagnetic wave incident normally on a thin metal film of thickness $h$ which is smaller than skin-depth $\delta=\sqrt{\frac{2}{\mu_{0} \sigma_{m} \omega}}$, but larger the characteristic thickness $h_{0}=\frac{2 \epsilon_{0} c}{\sigma_{m}} ;\left(h_{0} \ll h \leq \delta\right)$, shown in Fig. 7 (A). Here, $\sigma_{m}$ denotes the conductivity of the metal, $\mu_{0}$ denotes the magnetic permeability in vacuum and $\omega$ denotes the angular frequency of electromagnetic wave. The thickness at which the absorption loss by metal is $50 \%$, is known as the characteristic thickness $\left(h_{0}\right)$. For a metal with conductivity $10^{7} \Omega^{-1} \mathrm{~m}^{-1}$, the characteristic thickness is $0.53 \mathrm{~nm}$. At $1 \mathrm{THz}$, the skin-depth is about $100 \mathrm{~nm}$ or more for good metals. Therefore, the thickness range for transitional metal film is $5-100 \mathrm{~nm}$. The electric field amplitude transmission $(t)$ and reflection
A

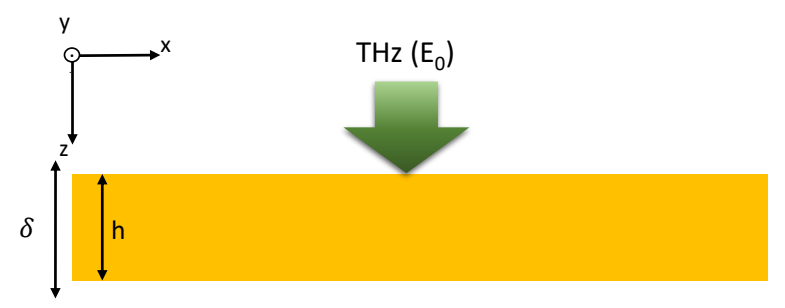

B

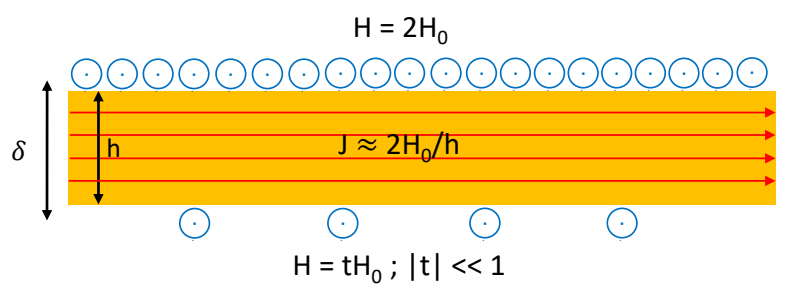

C

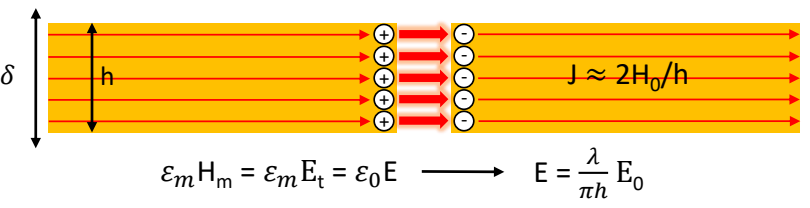

Figure 7. Field enhancement in metal film of thickness below skin-depth. (A) THz field incident normally on a metal film of thickness less than THz skin-depth. (B) Magnetic field of light (represented by blue encircled dots) and current density (represented by red lines) near the thin metal film. At incident surface (up), the magnetic field is twice that of the incident field and a transmission side (down), the magnetic field is much smaller as compared to the incident side. (C) Displacement current and $E$ around metal gap. Adapted with permission from Ref. ${ }^{23}$. ( ) 2018, De Gruyter.

coefficient $(r)$ for such thin films are given by

$$
t=\left|\frac{E_{t}}{E_{0}}\right| \approx \frac{2 \epsilon_{0} c}{\sigma_{m} h}=\frac{h_{0}}{h} \ll 1 ; r \approx 1-\frac{h_{0}}{h} \approx 1
$$

where, $E_{t}$ denotes the transmitted electric field. ${ }^{23}$

Considering a transverse magnetic polarized light $\left(\mathrm{E}_{x}, \mathrm{H}_{y}\right)$ is incident normally on a thin metal film. Due to reflection from the incident surface, the magnetic field of the light near the incident surface becomes twice that of the incident magnetic field. But the magnetic field on the transmission side is much smaller than the incident field (Fig. 7 (B)). Assuming that a constant electric field/current density is developed inside the thin film, using Ampere's law, the expression of the current density inside the thin film is $J=\frac{2 H_{0}}{h}$ (neglecting vacuum displacement current). ${ }^{23}$ Here, $J$ denotes the current density developed inside the thin metal film and $H_{0}$ denotes the incident magnetic field. The tangential component of electric fields at the air-metal interface are continuous and is given by

$$
E_{t}=E_{m}=\frac{J}{\sigma_{m}}=\frac{2 H_{0}}{\sigma_{m} h}=\frac{2 \epsilon_{0} c}{\sigma_{m} h} E_{0}
$$

therefore, reproducing Eq. 4. At transmitting side, electric field just inside the metal surface is denoted as $E_{m}$. 
A

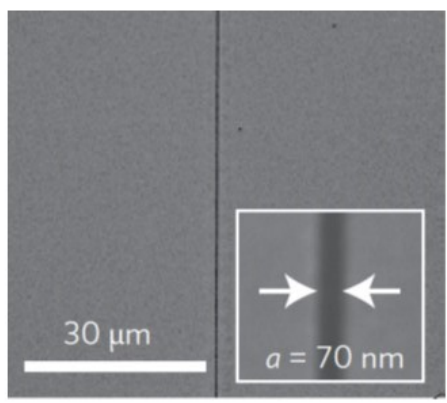

B
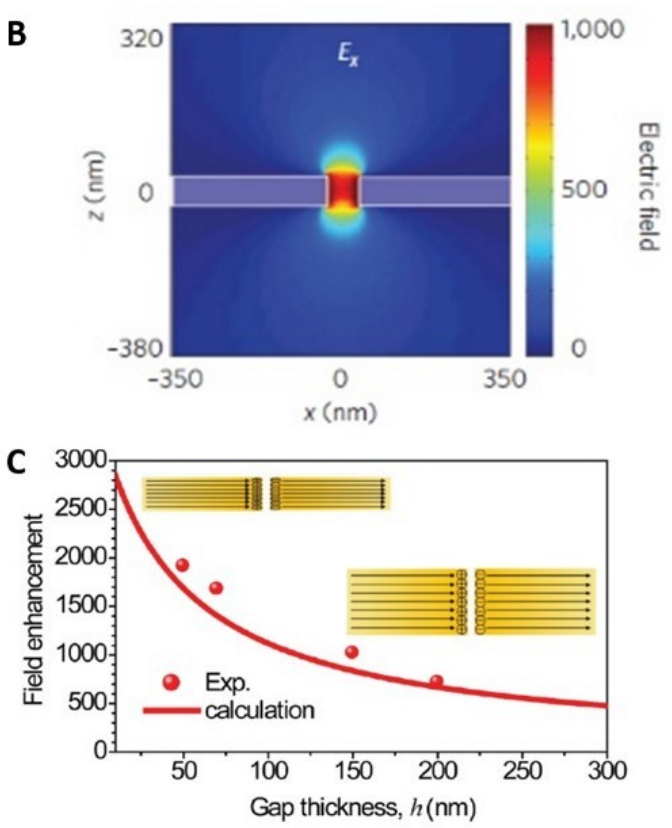

Figure 8. THz field enhancement in metal nanogaps at subskin depth regime. (A) SEM of a $70 \mathrm{~nm}$ wide gold nanogap fabricated using focused ion beam. (B) Horizontal electric field enhancement in a $70 \mathrm{~nm}$ gap, obtained from FDTD simulation. Reprinted with permission from Ref. ${ }^{34}$. (C) 2009, Springer Nature. (C) Variation of electric field enhancement with respect to gap thickness, indicating the $1 / h$ dependence of the field enhancement. Adapted with permission from Ref. ${ }^{16}$. (C) 2017, American Physical Society.

Across the air nanogap, the normal component of the displacement current will be same (Fig. 7 (C)), therefore electric field inside the air nanogap will be

$$
E=\left|\frac{\epsilon_{m}}{\epsilon_{0}}\right| E_{m} \approx \frac{\sigma_{m}}{\epsilon_{0} \omega} \frac{2 \epsilon_{0} c}{\sigma_{m} h} E_{0}=\frac{\lambda}{\pi h} E_{0}
$$

where, $\epsilon_{m}$ denotes the terahertz metal dielectric constant given by

$$
\epsilon_{m}=\epsilon_{\infty}+i \frac{\sigma_{m}}{\omega\left(1-i \frac{\omega}{\gamma}\right)} \approx i \frac{\sigma_{m}}{\omega}
$$

where, $\epsilon_{\infty}$ denotes the dielectric constant of metal at high-frequency and $\gamma$ denotes the Drude damping constant.

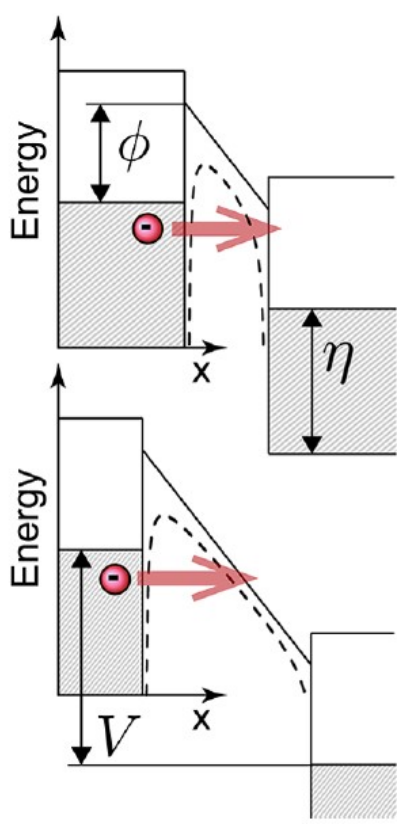

Figure 9. Electron tunnelling through thick and thin barriers. Due to the high gap electric field $E$, separation of Fermi level $\eta$ in two metals by potential energy $V=e E w$ takes place. $\phi$ represents the rectangular barrier height and the dotted line describes the barrier including image forces. Reprinted with permission from Ref. ${ }^{24}$. (c) 2015, American Physical Society.

Through this analogy, it can be said that with an increase in the conductivity of the metal, weaker electric fields are induced inside the metal. This is compensated by the metal's high dielectric constant when the displacement boundary condition is considered. Therefore, the field enhancement is independent of the conductivity of the metal, which is mathematically proven above. Fig. 8 (A) is the SEM of a gold nanogap sample of gap width $70 \mathrm{~nm}$, fabricated by Seo et al. ${ }^{34}$ using a focused ion beam technique. Fig. 8 (B) shows the x-z plane horizontal electric field enhancement in $70 \mathrm{~nm}$ gap, analyzed from FDTD simulation at $0.1 \mathrm{THz}$. In the figure, it can also be seen that the electric field does not penetrate into the metal, rather it is seen to be completely concentrated inside the nanogap, even if the gap size is much smaller than the skin depth $(250 \mathrm{~nm})$. Fig. 8 (C) shows the varying electric field enhancement of $5 \mathrm{~nm}$ gap with respect to the gap thickness $(h)$ at a frequency of $0.3 \mathrm{THz}$. The red dots signify the experimental data and the red solid line shows the calculated data from the modal expansion. the figure also signifies the $1 / h$ dependence of the field enhancement.

\section{B. THz Field Enhancement in Sub-Nanometer and Ångstrom Metallic gaps: Quantum Regime}

Recently, Bahk et al. ${ }^{16}$ discussed about the field enhancement in thin $(w \sim h<\delta)$ and thick $(w<\delta<h)$ 
A

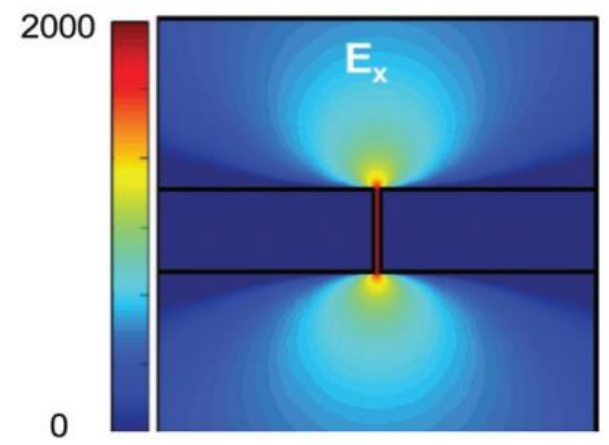

B

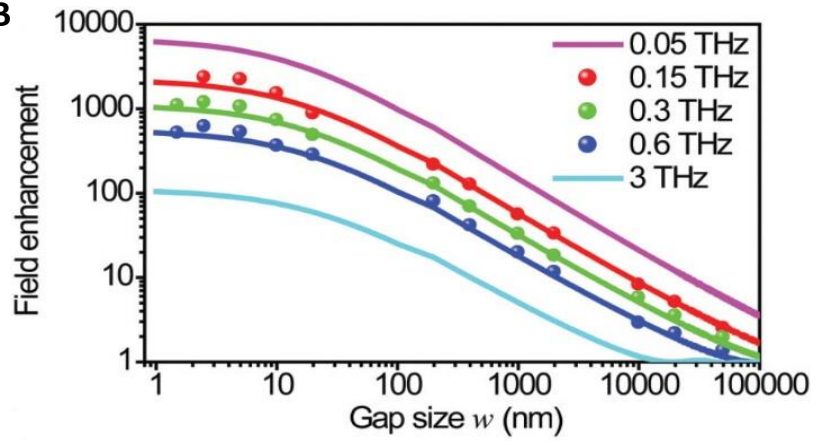

Figure 10. Classical limit of THz field enhancement before entering quantum regime inside gold nanogap. (A) FDTD simulated horizontal electric field around gold nanogap of width $1.5 \mathrm{~nm}$. (B) Variation of field enhancement in different gap widths for various $\mathrm{THz}$ frequencies. Solid lines represents the theoretically calculated values from modal expansion formalism. Adapted with permission from Ref. ${ }^{16}$. (C) 2017, American Physical Society.

narrow gaps, and in wide $(\delta, h<w)$ gaps. In wide gaps, the charges are mostly spread over the surface outside the gap rather than at metal edges (Fig. 6 (B)), which results in a decrease of field enhancement. In narrow gaps, charges mostly accumulate at the metal edges of the gap (Fig. $6(\mathrm{C})$ ), resulting in stronger field enhancement. As the width of the gap $(w)$ decreases further entering the sub-nanometer and Ångstrom regime, the charge distribution becomes insensitive to gap size and electron tunnels through the potential barrier of the nanogap, showing quantum effects. ${ }^{197,199-210}$ When $\mathrm{THz}$ electromagnetic waves are incident on the nanogap, a transient voltage is developed in the dielectric gap which bends the conduction band of the dielectric toward the Fermi energy of metals (Fig. 9). ${ }^{24}$ This increases the chances for electron tunneling through the potential barrier, causing non-linear transmissions.

Bahk et al. also investigated the classical limit of field enhancement before the gap-width entered the quantum regime. ${ }^{16}$ They reported that the field enhancement exhibited saturation behavior rather than a monotonous increasing nature. This showed that the nanogap acts like a charged capacitor ${ }^{34}$ which is shown in the Fig. 6 (A), whose total induced charge is inversely proportional to the frequency of a light-induced alternating current of THz-frequency. FDTD analysis of the electric field distribution around $1.5 \mathrm{~nm}$ wide gap in 150 $\mathrm{nm}$ thick gold film at a frequency of $1.5 \mathrm{THz}$, as shown in Fig. 10 (A). As expected the horizontal electric field is strongly concentrated and is enhanced by a factor of 2000 inside the gap. Fig. 10 (B) shows the electric field enhancement in different gap size for various frequencies. The theoretical calculation of the modal expansion for the perfect electric conductor (PEC) model is shown by solid lines.

\section{The Finite-Difference Time-Domain (FDTD) Algorithm : A Computational Electromagnetism Approach}

The finite difference time domain (FDTD) method ${ }^{211}$ is used to solve Maxwell's equations in the time domain and generally used for computational electrodynamics.

$$
\begin{gathered}
\vec{\nabla} \cdot \vec{D}=\rho \\
\vec{\nabla} \cdot \vec{B}=0 \\
\vec{\nabla} \times \vec{E}=-\frac{\partial \vec{B}}{\partial t} \\
\vec{\nabla} \times \vec{H}=\vec{J}+\frac{\partial \vec{D}}{\partial t}
\end{gathered}
$$

are the four Maxwell's equations where, the electric field, magnetic field, electric displacement field, magnetic flux density, free electric charge density, and free current density are denoted by E, H, D, B, $\rho$ and J, respectively. ${ }^{212,213}$ These equations are solved numerically on a discrete grid in both space and time, and derivatives are handled with finite differences. No approximations or assumptions are made about the system, making this method highly versatile and accurate. It is a fully vectorial simulation method as it solves for all electric and magnetic field vector components. Being a time-domain method, FDTD can be used to calculate broadband results from a single simulation. FDTD method is typically used when the feature size is of the order of the wavelength. It is general, versatile, accurate, broadband and fasts which makes it the most reliable method in computational electrodynamics.

FDTD algorithm solves Maxwell's curl equations in non-magnetic materials.

$$
\frac{\partial \vec{D}}{\partial t}=\nabla \times \vec{H}
$$

where, $\vec{D}=\epsilon \vec{E}$. Since the region is vacuum, $\vec{J}=0$.

$$
\vec{D}(\omega)=\epsilon_{0} \epsilon_{r}(\omega) \vec{E}(\omega)
$$



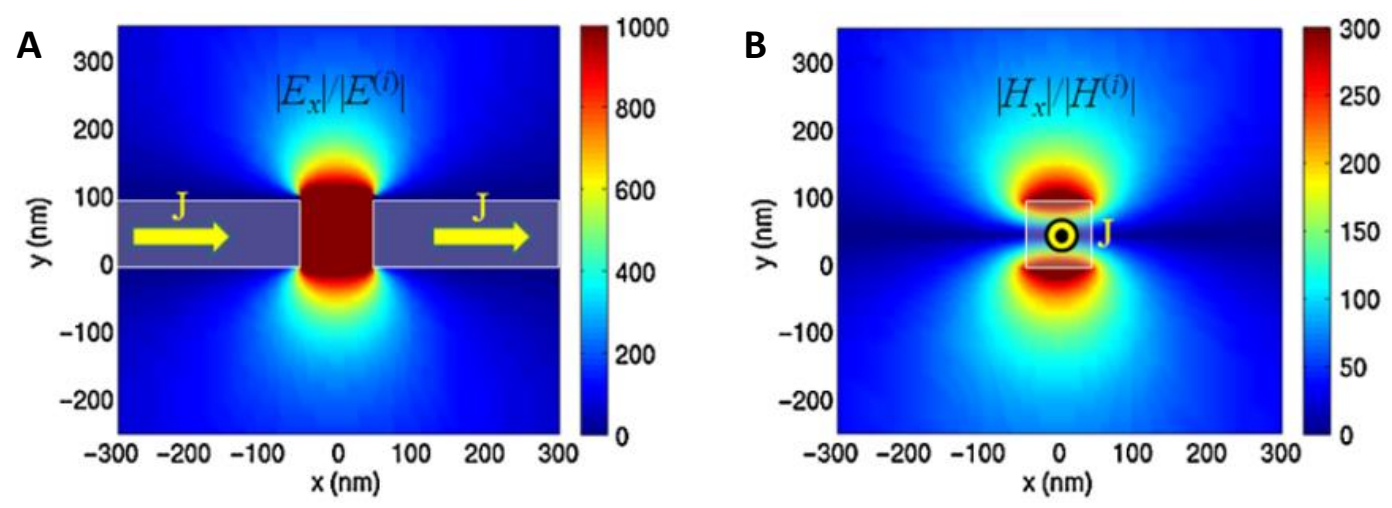

Figure 11. FDTD analysis of a gold nanogap and nanorod. (A) Horizontal electric field around $100 \mathrm{~nm}$ gold nanogap. (B) Simulated magnetic field around a gold nanorod. Yellow arrows represent the current flow. Reprinted with permission from Ref. $^{145}$. (C) 2009, American Physical Society.

$$
\frac{\partial \vec{H}}{\partial t}=-\frac{1}{\mu_{0}} \nabla \times \vec{E}
$$

with $\mathrm{H}, \mathrm{E}$, and $\mathrm{D}$ describing the magnetic field, electric field and displacement field, respectively, while $\epsilon_{0}(\omega)$ is a complex relative dielectric constant given by

$$
\epsilon_{r}(\omega)=n^{2}
$$

, where $\mathrm{n}$ denotes refractive index of the material. In 3-D, the six electromagnetic components of Maxwell's equations are $-E_{x}, E_{y}, E_{z}$ and $H_{x}, H_{y}, H_{z}$. Assuming that in z-dimension, the structure is infinite and the fields are independent of $\mathrm{z}$, such that

$$
\begin{gathered}
\epsilon_{r}(\omega, x, y, z)=\epsilon_{r}(\omega, x, y), \text { and } \\
\frac{\partial \vec{E}}{\partial z}=\frac{\partial \vec{H}}{\partial z}=0
\end{gathered}
$$

then, two independent sets of Maxwell's equations will be created. Each set will contain three vector quantities which can only be solved in $x-y$ plane. The equation containing the components - $E_{x}, E_{y}, H_{z}$, is known as transverse electric (TE) equation, and the equation containing the components - $H_{x}, H_{y}, E_{z}$, is known as transverse magnetic (TM) equation. ${ }^{211}$ Therefore, in TM case, Maxwell's equations reduces to

$$
\begin{gathered}
\frac{\partial D_{z}}{\partial t}=\frac{\partial H_{y}}{\partial x}-\frac{\partial H_{x}}{\partial y} \\
D_{z}(\omega)=\epsilon_{0} \epsilon_{r}(\omega) E_{z}(\omega) \\
\frac{\partial H_{x}}{\partial t}=-\frac{1}{\mu_{0}} \frac{\partial E_{z}}{\partial y} \\
\frac{\partial H_{y}}{\partial t}=-\frac{1}{\mu_{0}} \frac{\partial E_{z}}{\partial x}
\end{gathered}
$$

and for TE case, Maxwell's equations reduces to

$$
\begin{gathered}
\frac{\partial H_{z}}{\partial t}=-\frac{1}{\mu_{0}}\left[\frac{\partial E_{y}}{\partial x}-\frac{\partial E_{x}}{\partial y}\right] \\
D_{x}(\omega)=\epsilon_{0} \epsilon_{r}(\omega) E_{x}(\omega) \\
D_{y}(\omega)=\epsilon_{0} \epsilon_{r}(\omega) E_{y}(\omega) \\
\frac{\partial D_{x}}{\partial t}=\frac{\partial H_{z}}{\partial y} \\
\frac{\partial D_{y}}{\partial t}=\frac{\partial H_{z}}{\partial x}
\end{gathered}
$$

These equations are solved on a discrete, spatial and temporal grid. Each field component is solved at a slightly different location within the grid cell (or, Yee cell). ${ }^{211}$

With this method we can simulate the electric and magnetic fields around any nanostructures using commercially available softwares like, FDTD Solutions (Lumerical Inc., Canada), COMSOL, etc. Fig. 11 shows the FDTD analysis of gold nanogap (100 nm thick and wide gap) and nanorod, respectively. ${ }^{145}$

\section{THZ-TDS AND SENSING OF MOLECULES AND BIOMOLECULES}

In the late 1980s, Grischkowsky et al. ${ }^{214-216}$ introduced the terahertz time-domain spectroscopy technique (Fig. 13). It is a spectroscopic technique in which short- $\mathrm{THz}$ pulses are used to probe the properties of matter. The system generally consisted of the femtosecond laser (fs-laser), which operates at a repetition rate of $100 \mathrm{MHz}$, producing a $100 \mathrm{fs}$ laser pulse train. Using a beam splitter, the fs-pulse train is split into two 


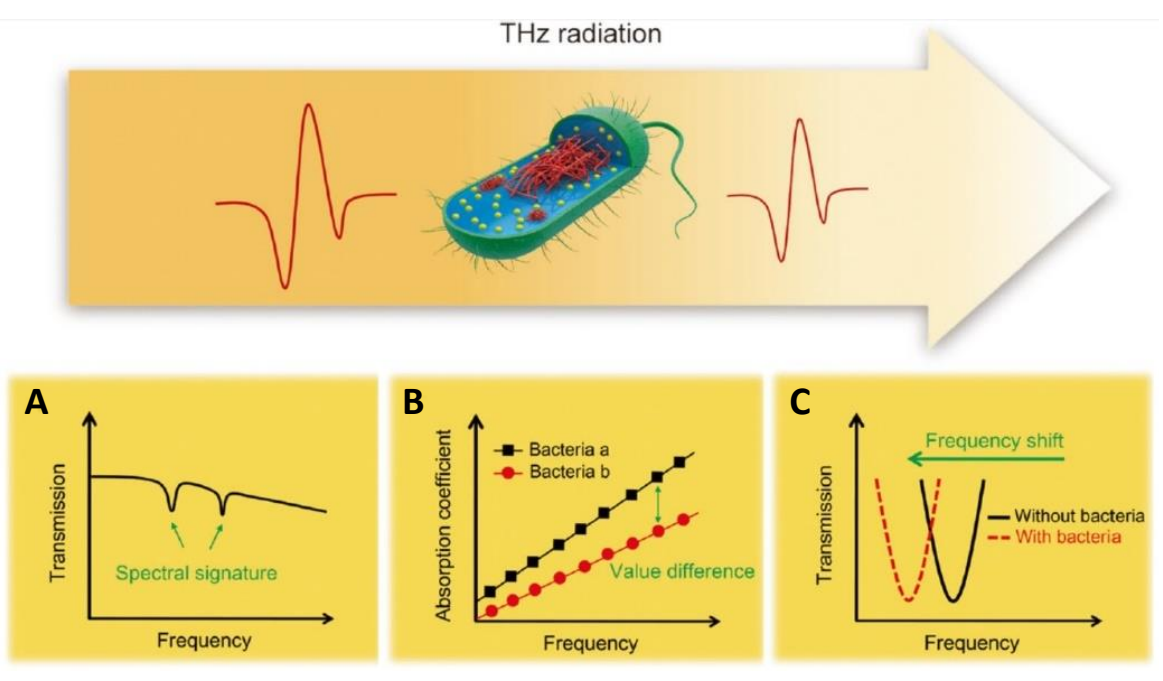

Figure 12. Spectral analysis for identification of biomaterials. (A) Distinct spectral signatures can be used to discriminate a bacterial cell, spore and intracellular metabolite. (B) Bacterial species can be differentiated from their differences in THz optical constants. (C) Resonant frequency shift analysis using $\mathrm{THz}$ nanoantenna biosensors can be used to study the presence of biomaterials. Adapted with permission from Ref. ${ }^{87}$. (C) 2016, Springer Nature.

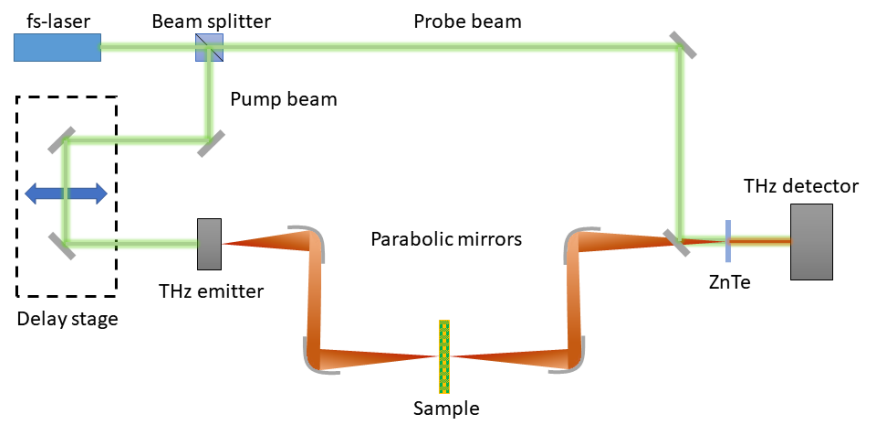

Figure 13. Conventional THz time-domain spectroscopy setup developed by Grischkowski et al. Adapted with permission from Ref. ${ }^{145}$. (c) 2016, Springer Nature.

beams: a pump beam and a probe beam. The pump beam is made to be incident on a $\mathrm{THz}$ emitter to emit $\mathrm{THz}$ pulses, which is collimated to the sample using a pair of parabolic mirrors. Concurrently, the probe beam is used in a time-gated manner for the detection of $\mathrm{THz}$ electric field which contains time-domain information of phase and amplitude. Coherently, the transmitted $\mathrm{THz}$ electric field is measured as a function of time to obtain a time-domain signal which is converted to noise-free frequency-domain signal using Fourier transformation function.

THz-TDS is a non-contact, non-invasive and labelfree detection technique and is extensively applied in biomedical imaging. However, THz-TDS has proved to be less sensitive for detection of micro-organisms (like yeast, molds, and bacteria) of size equal to or less than $\lambda / 100$, due to their transparency under $\mathrm{THz}$ frequencies. Recently, plasmonic nanoantennas ${ }^{88,89,217}$ and metamaterials ${ }^{92-94}$ have proved to be effective devices for detection of micro-organisms, organic- and biomolecules. These devices show resonances with strong field enhancement across the gap in the $\mathrm{THz}$ frequency range, which is highly sensitive to changes in dielectric constant of the gap region. Therefore, these devices are used as biosensors for ultra-sensitive and label-free detection of micro-organisms, organic- and biomolecules. In this section, we discuss the two widely used label-free THz-TDS methods : (i) THz-TDS using molecular dynamic (MD) simulations, and (ii) THz sensing by nanoantennas and metamaterials.

\section{A. Computational THz-TDS using Molecular Dynamics (MD) Simulation}

MD simulation is a computational method used to study the dynamics of a system of molecules in the condensed phase. To accurately study the dynamics of a complex molecule, one needs to employ the quantum model to study the wave function of each sub-atomic particle. But MD employs classical Newtonian mechanics to study the dynamics of the system, which makes it less accurate. Specifically, it integrates Newton's equation of motion in discrete time-steps. Due to high $\mathrm{THz}$ absorption by water, THz-TDS becomes challenging for water-based systems. Therefore, MD simulation is one of the preferred methods to study the water dynamics of a hydrated molecular system. In MD, water dynamics has a great significance in the structural arrangements of molecules, which occurs because the molecule alters the dynamics of the surrounding water molecules, adopting a quasi-coherent character, caused by reorganized, loose 


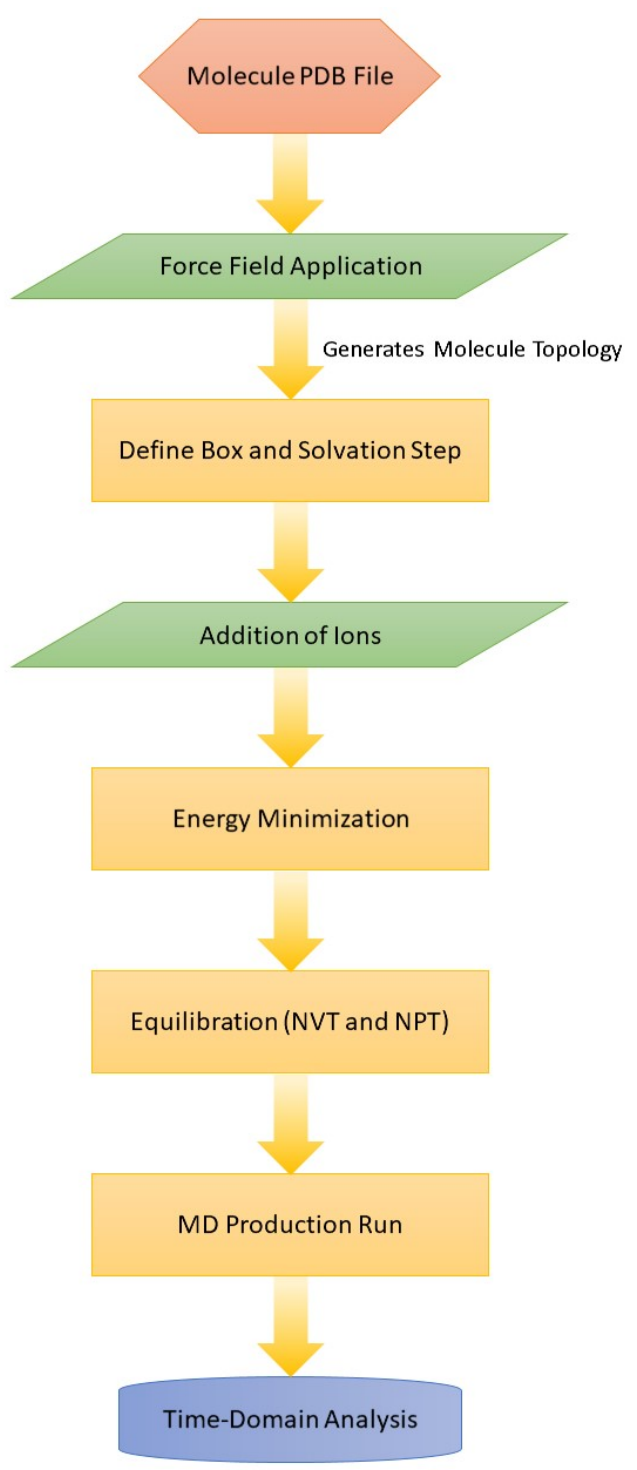

Figure 14. Flowchart of a general MD simulation process.

hydrogen-bond network.

A significant number of MD-based computational analysis have been performed to interpret the $\mathrm{THz}$ absorption spectra of protein solutions. Many studies showed the anomalous dynamics of water in the hydration shell due to the fact that surrounding water molecules were heterogeneously perturbed by the solute. ${ }^{20-224}$ Bandyopadhyay et al. have performed a significant amount of MD simulations to study the effects of hydration of protein molecules. ${ }^{223,225-232}$ Various methods have been used to study the thickness of villin headpiece sub-domain HP-36 which includes MSD, velocity autocorrelation function analysis, $\mathrm{H}-$ bond time correlation function analysis and reorientational correlation function analysis of water molecules. According to the simulation results, the different helical segments had a heterogeneous influence on the wa-
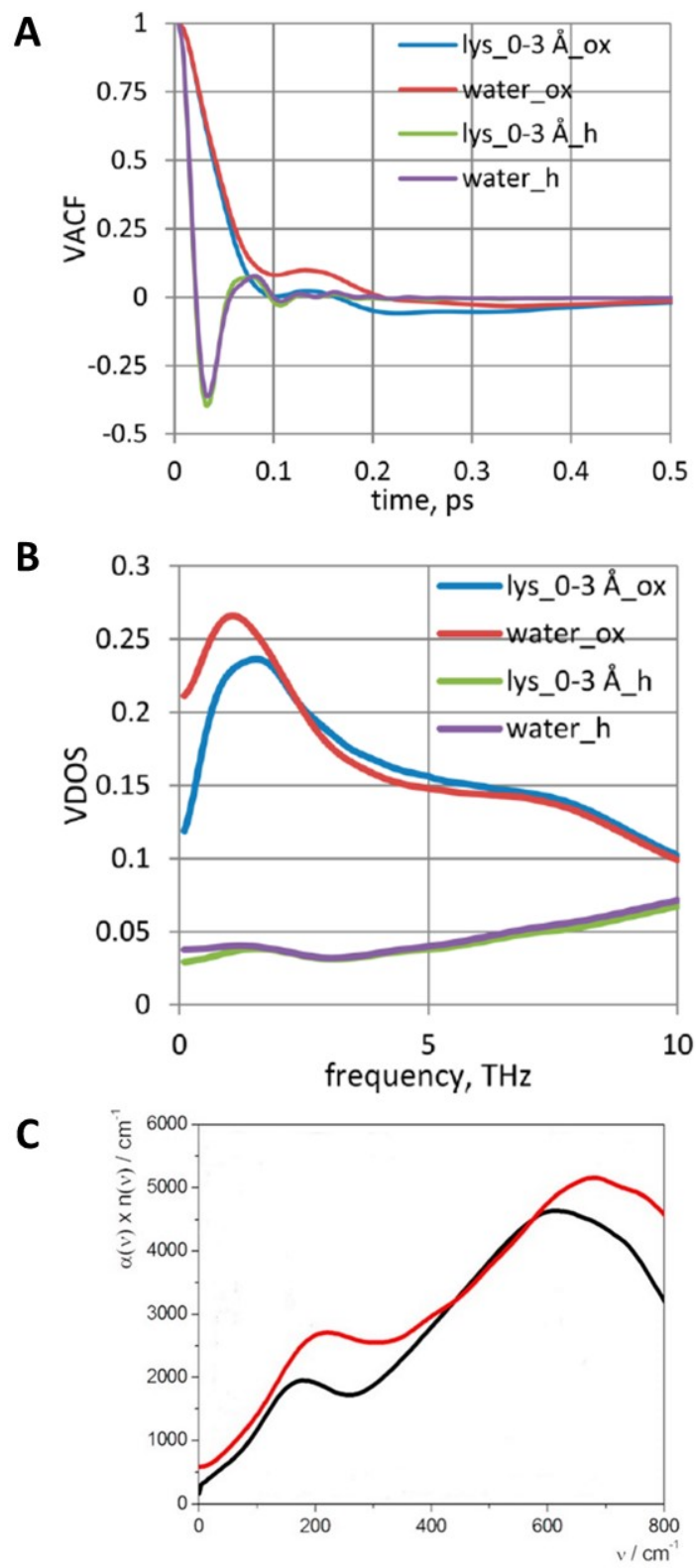

Figure 15. THz time-domain analysis obtained using MD simulations. (A) Velocity autocorrelation fuction (VACF) and (B) vibrational density of states of oxygen (ox) and hydrogen (h) in first solvation layer ( $3 \AA$ ) of lysosome and bulk water. Reprinted with permission from Ref. ${ }^{218}$. (C) 2013, American Chemical Society. (C) Absorption spectrum of water obtained experimentally (black line) and from MD simulation (red line). Adapted with permission from Ref. ${ }^{219}$. (C) 2014, American Chemical Society.

ter dynamics, which were limited to the first hydration layer. ${ }^{223}$ Some similar results were obtained in which it was demonstrated a clear distinct power spectrum (VDOS) for water molecules bonded to different planes of antifreeze protein by hydrogen in the spectral domain of 1-4 THz. ${ }^{224}$ While studying the vibrational spectrum of water in the villin headpiece subdomain hy- 
dration layer, $\mathrm{O} \cdots \mathrm{O} \cdots \mathrm{O}$ bending mode showed a blueshift in the first hydration layer, which is noticed for water molecules bonded to protein by hydrogen. ${ }^{225}$ It also showed the possibility of the structural flexibility of protein. Later an atomistic MD simulation of hen egg-white lysosome solvated in explicit water molecules at room temperature was performed, ${ }^{226}$ which reported that a few large-amplitude bistable motions exhibited by two coils controlled the overall flexibility of protein molecules. A series of MD simulations were performed to compare the experimental data of far-infrared spectroscopy used to study the dynamics of three aqueous peptides with varied helicity. ${ }^{233}$ Using the first principles, extensive studies on water, resolved in time and space, reported that the group motion of H-bonded molecules in the second solvation shell significantly contributed to the absorption at about $2.4 \mathrm{THz}$, also showing the presence of third-shell effects. ${ }^{234}$ Heyden et al. in their future publication ${ }^{235}$ showed the capability of nonpolarizable water models in reproducing lowfrequency, inter-molecular vibration of water since electronic polarization is dominated by the static molecular dipoles. The depth of hydration shell in the hydrated lysosome, BPTI, TRP-cage, and TRP-tail was estimated using MD simulations. ${ }^{218}$

To perform a basic MD simulation of a molecule, ${ }^{236}$ first and foremost a force field must be applied to characterize the molecular interaction of the molecules. In the next step, the molecule is positioned in a unit cell of desired shape and size followed by the addition of water molecules, known as solvation step. Then ions are added to neutralize the net charge of the system. After the addition of ions, the system is then relaxed through a process known as energy minimization. This process also ensures that the system is free from steric clashes or inappropriate geometry. For further simulation, the system needs to be brought to the desired temperature. After attaining the desired temperature, an adequate amount of pressure must be applied until it reaches a proper density. This whole process is known as equilibration and is conducted in two phases. The first phase is conducted to attain the desired temperature under NVT (constant Number of particles, Volume, and Temperature) or isothermal-isochoric ensemble. The second phase is conducted to stabilize both pressure and density of the system under NPT (constant Number of particles, Pressure, and Temperature) or isothermal-isobaric ensemble. After attaining the desired temperature and pressure, the system is finally ready for the MD simulation, known as MD production run. After performing the final MD-run, the system is ready to be analyzed. From MD simulation, the time-dependent properties are obtained via. correlation function, like velocity autocorrelation function (VACF). The time-domain properties are then converted to frequency-domain properties using Fourier transformation function, for example, the vibrational density of states (VDOS). The whole MD sim-

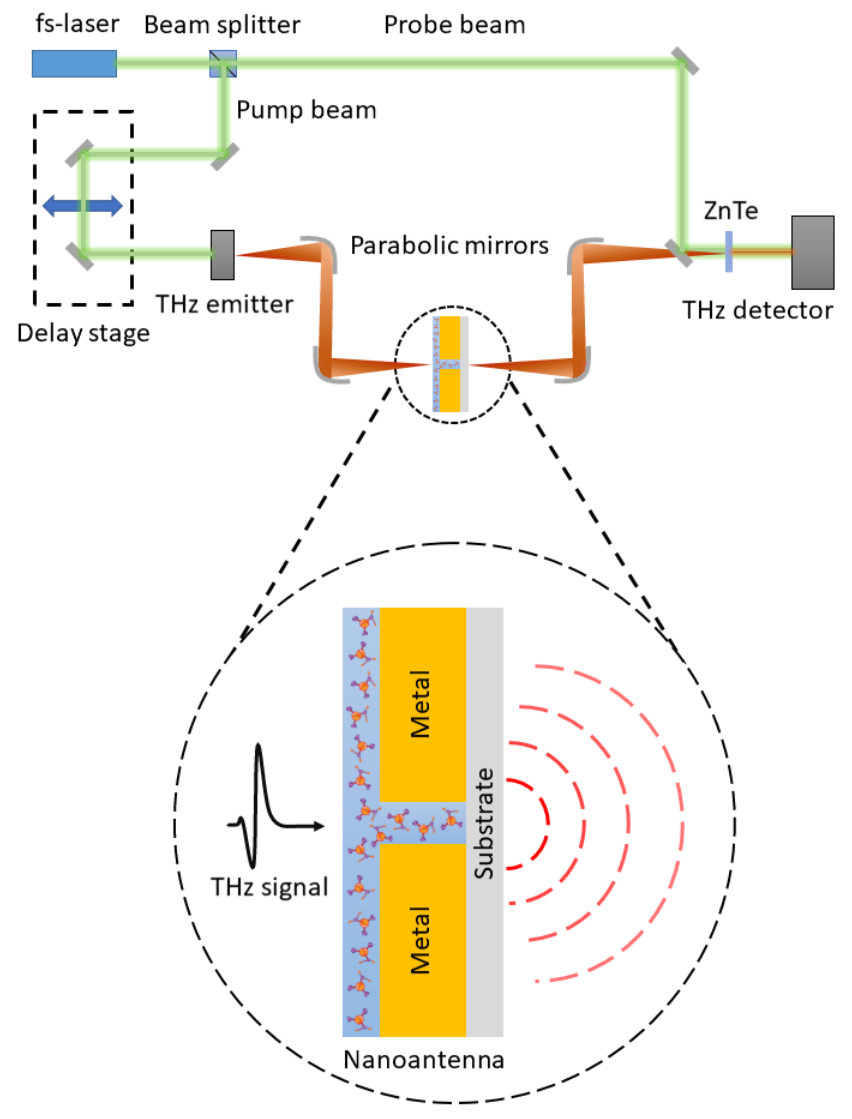

Figure 16. Application of $\mathrm{THz}$ nanoantenna in developing a highly-sensitive THz-TDS technique.

ulation steps are summed up in a flowchart (Fig. 14).

Fig. 15 (A) shows the MD simulation generated VACF curves for oxygen and hydrogen atoms in bulk water and in the water within $3 \AA$ of lysosome molecule (first hydration layer). The figure depicts two results: i) The hydrogen dynamics get uncorrelated faster $(0.85$ ps earlier) than oxygen dynamics, and ii) The dynamics of the oxygen atoms are much more restricted in the first hydration layer than in bulk water (as compared from their extremums) due to caging-effect. The corresponding VACF data was further Fourier transformed to get a set of VDOS data as shown in Fig. 15 (B). In the figure, at $1.1 \mathrm{THz}$ a well-defined peak is shown by the oxygen atoms of bulk water signifying the bending motion of $\mathrm{O}-\mathrm{O}-\mathrm{O}$ atoms (known as triplets of H-bonded oxygen atoms). But in the case of lysosome-bounded water molecules, this peak is blue-shifted by $0.4 \mathrm{THz}$ signifying that the $\mathrm{H}$-bonds between $\mathrm{O}$ atoms gets stronger in the hydration layer. This peak has a lower amplitude compared to that of the oxygen atoms of bulk water, as the presence of lysosome does not have much influence on the vibrational mode of water molecules. The VDOS of hydrogen in both situations is very flat as they are not much influenced by the protein molecule. The absorption spectrum can be solved by applying Fourier trans- 
A

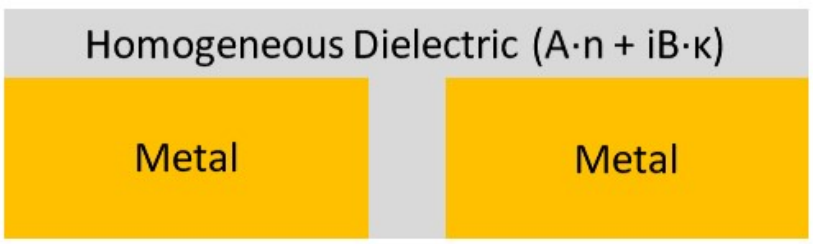

B

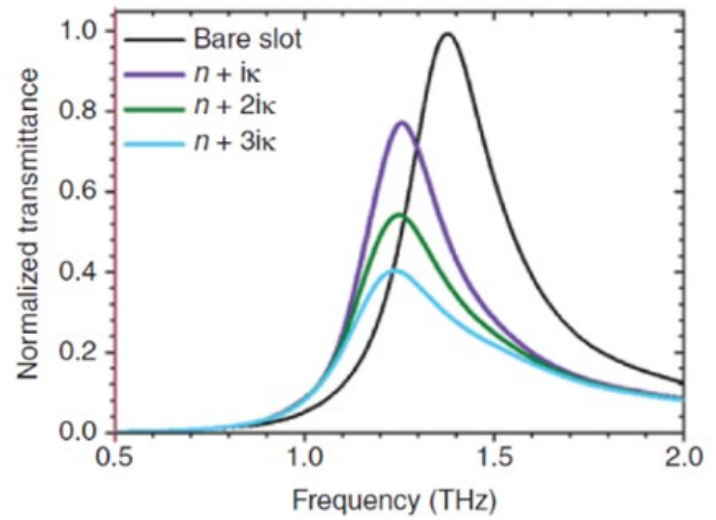

C

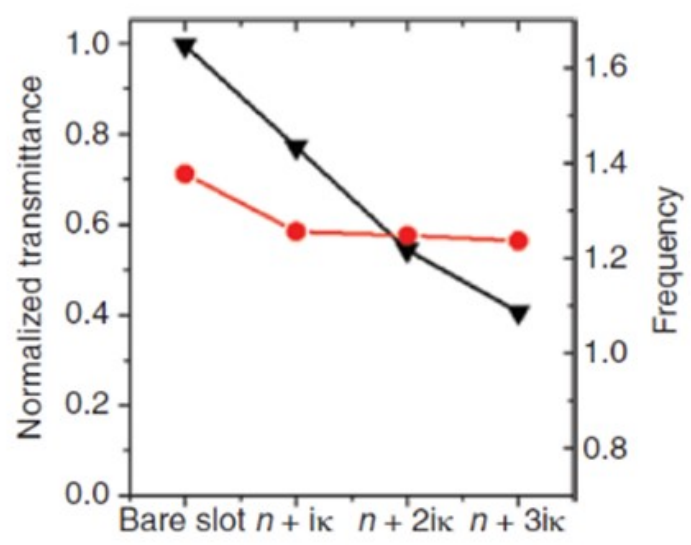

Figure 17. Interpretation of nanoantenna enhanced $\mathrm{THz}$ interactions of biomaterials using FDTD method. (A) Schematic of the cross section view of a metallic nanogap cladded with a absorptive homogeneous dielectric film with complex refractive index. (B) Normalized transmittance simulated by varying the imaginary part of the complex refractive index of the homogeneous dielectric film. (C) Transmittance peak values and resonance frequencies are plotted for various complex refractive indices. Adapted with permission from Ref. ${ }^{23}$. (c) 2018, De Gruyter.

form on total dipole moment autocorrelation function of the system computed from MD simulation, ${ }^{219}$ given by

$$
\alpha(\omega)=\frac{1}{4 \pi \epsilon_{0}} \frac{2 \pi \omega^{2}}{3 V k_{B} T c n(\omega)} \int_{0}^{\infty} e^{i \omega t}\langle M(0) M(t)\rangle d t
$$

here, $\mathrm{V}$ denotes the volume of the system, $\mathrm{k}_{B}$ denotes the Boltzmann's constant, $\mathrm{T}$ denotes the absolute temperature of the system, $\mathrm{c}$ denotes the speed of light and $\mathrm{n}(\omega)$ denotes the frequency dependent refractive index.
A

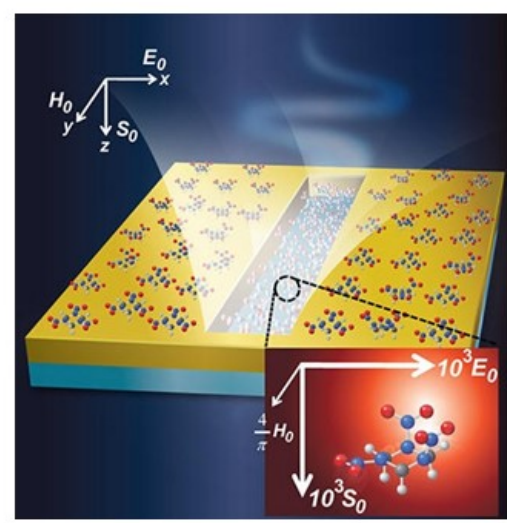

B
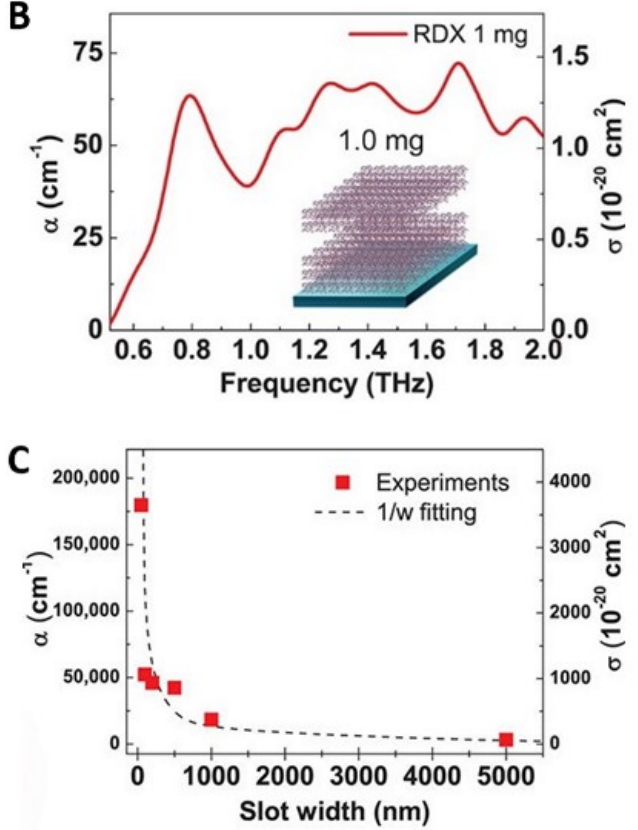

Figure 18. Effects of various gap-widths on THz-sensing of RDX molecules. (A) Schematic of the cross section of a THz single-slot nanoantenna containing RDX molecules. (B) Absorption spectrum of RDX molecules $(1 \mathrm{mg})$ onto the bare quartz substrate. (C) Absorption coefficients peaks and cross section of RDX molecules in various nanoslot gap-widths $w$. The black dashed line signifies the $1 / w$ dependence. Reprinted with permission from Ref. ${ }^{89}$. (C) 2013, American Chemical Society.

Figure $15(\mathrm{C})$ is the absorption spectra of bulk water obtained experimentally (black line) ${ }^{237}$ and by MD simulation (red line), ${ }^{238}$ describing the inter-molecular hydrogen bond collective modes.

\section{B. THz-Nanoantenna and Metamaterials Sensing of Micro-organisms, Organic- and Biomolecules}

$\mathrm{THz}$ nano-structures can be used for ultra-sensitive, label-free and non-invasive sensing of molecules and 

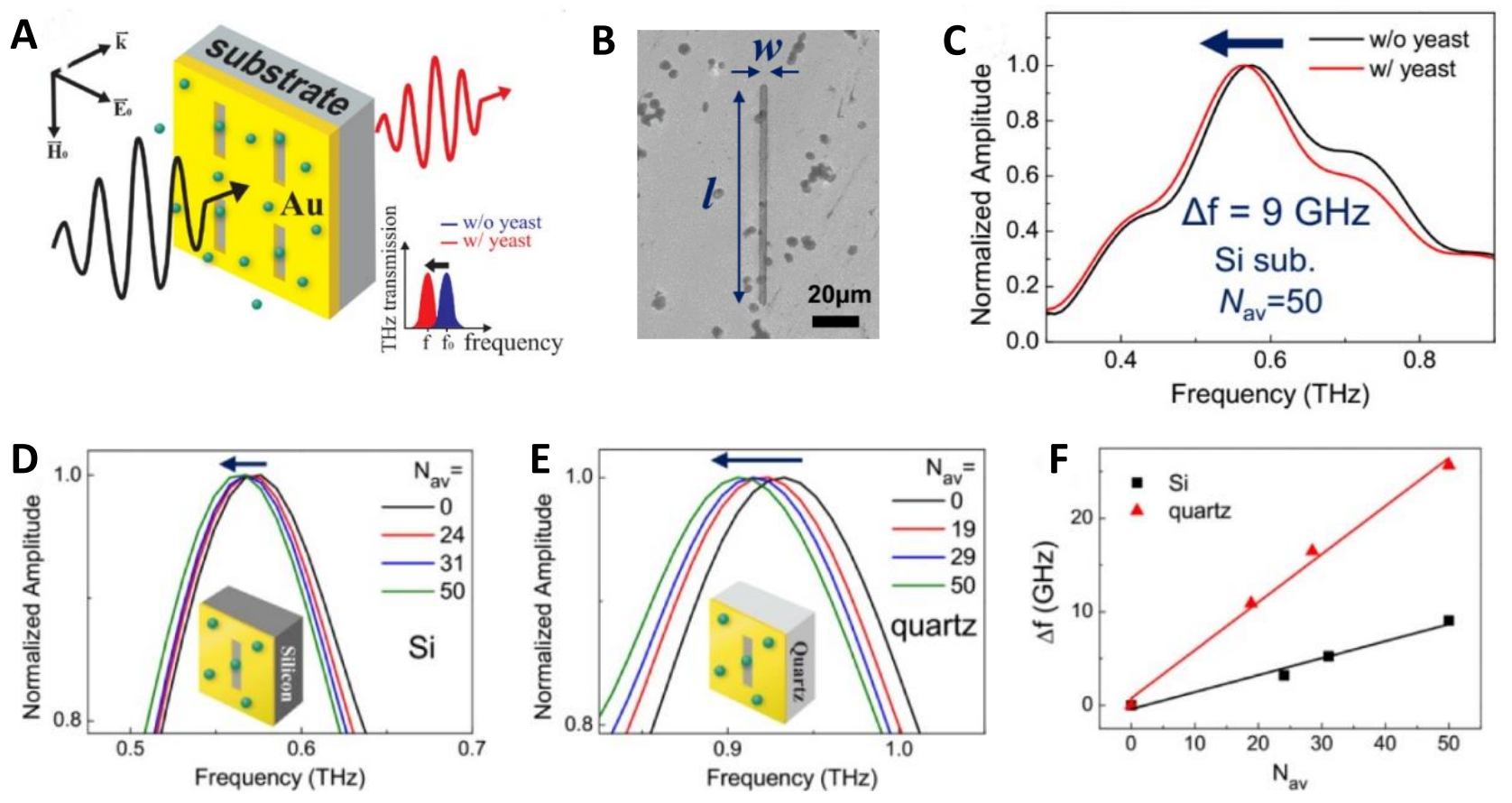

Figure 19. Effects of different substrates on yeast detection. (A) Schematic of THz sensing of yeast cells using a THz slot antenna. (B) SEM image of yeast cells deposited on $2 \mu \mathrm{m}$ wide and $100 \mu \mathrm{m}$ long slot antenna. (C) $\mathrm{THz}$ transmission amplitudes for THz slot antenna on Si substrate showing a red-shift in the resonant frequency of the slot antenna due to the presence of yeast cells. $\mathrm{THz}$ transmission amplitudes for $\mathrm{THz}$ slot antenna on (D) Si- and (E) quartz-substrates at different yeast cell concentration ( $\mathrm{N}_{a v}$ ). (F) Shifts in resonance frequency of $\mathrm{THz}$ slot antenna on $\mathrm{Si}$ - and quartz-substrates with respect to $\mathrm{N}_{a v}$. Reprinted with permission from Ref. ${ }^{93}$. (C) 2014, Springer Nature.

biomaterials. As discussed earlier in this section, the strong $\mathrm{THz}$ field enhancement along with resonances in the gap region of these nano-structures are sensitive to the changes in the dielectric constant of the insulating gap material. If the gap is filled with desired biomaterial sample, due to enhanced THz-interaction, the dielectric (i.e., the biomaterial sample) will show an enhanced $\mathrm{THz}$-absorption therefore, the THz optical properties can be studied from the $\mathrm{THz}$ dielectric response. Thereby, making the necessary change in the THz-TDS setup (Fig. 16), enables enhanced sensing of molecules and micro-organisms which were not sensed by conventional THz-TDS setup. Therefore, from the Fourier transformed spectra (frequency-domain amplitude and phase) obtained from the sample and reference transmitted signals, the complex optical properties ae computed from the following relation:

$$
A_{s}(\omega)=A_{r}(\omega) \cdot e^{-\left(\frac{d \cdot \alpha(\omega)}{2}\right)} \cdot e^{\left(i \frac{\omega}{c} n(\omega) d\right)}
$$

where, $\mathrm{A}_{s}$ and $\mathrm{A}_{r}$ are the amplitudes of sample and reference transmitted signals, respectively. The real parts of the absorption coefficient and the index of refraction are denoted by $\alpha(\omega)$ and $n(\omega)$, respectively, and thickness of the dielectric is denoted by $d$. The real part of refractive index ${ }^{87}$ is given by

$$
n(\omega)=1+\frac{\left(\phi_{r}-\phi_{s}\right) c}{2 \pi d f}
$$

and applying Beer-Lambert law, absorption coefficient ${ }^{23}$ is given by

$$
\alpha(\omega)=-\frac{2}{d} \ln (T)=-\frac{2}{d} \ln \left(\left(\frac{A_{s}(\omega)}{A_{r}(\omega)}\right)^{2}\right)=\frac{4 \pi f k}{c}
$$

where, $\phi_{r}$ and $\phi_{s}$ are the phases of sample and reference transmitted signals, respectively, $\mathrm{T}$ denotes the transmittance and $f$ is the frequency of the input THz-signal.

Through FDTD simulation, an intense study of this biosensing process can be done. The biomaterial sample film is assumed to be a homogeneous dielectric film of a thickness proportional to the molecular concentration, is sandwiched between two gold films (Fig. 17 (A)). The dielectric film has a complex refractive index $A \cdot n+$ $\mathrm{i} B \cdot \kappa$, where $\mathrm{A}$ and $\mathrm{B}$ are constants whose values range from 1.0 - 3.0 over a frequency band. The absorptive dielectric film was inserted in the gold nanogap by applying an auxiliary differential equation. To study the electric field interaction in the absorptive dielectric film, a non-uniform mesh was applied over the whole film with the smallest step-size of $10 \mathrm{~nm}$. To the nanoan- 

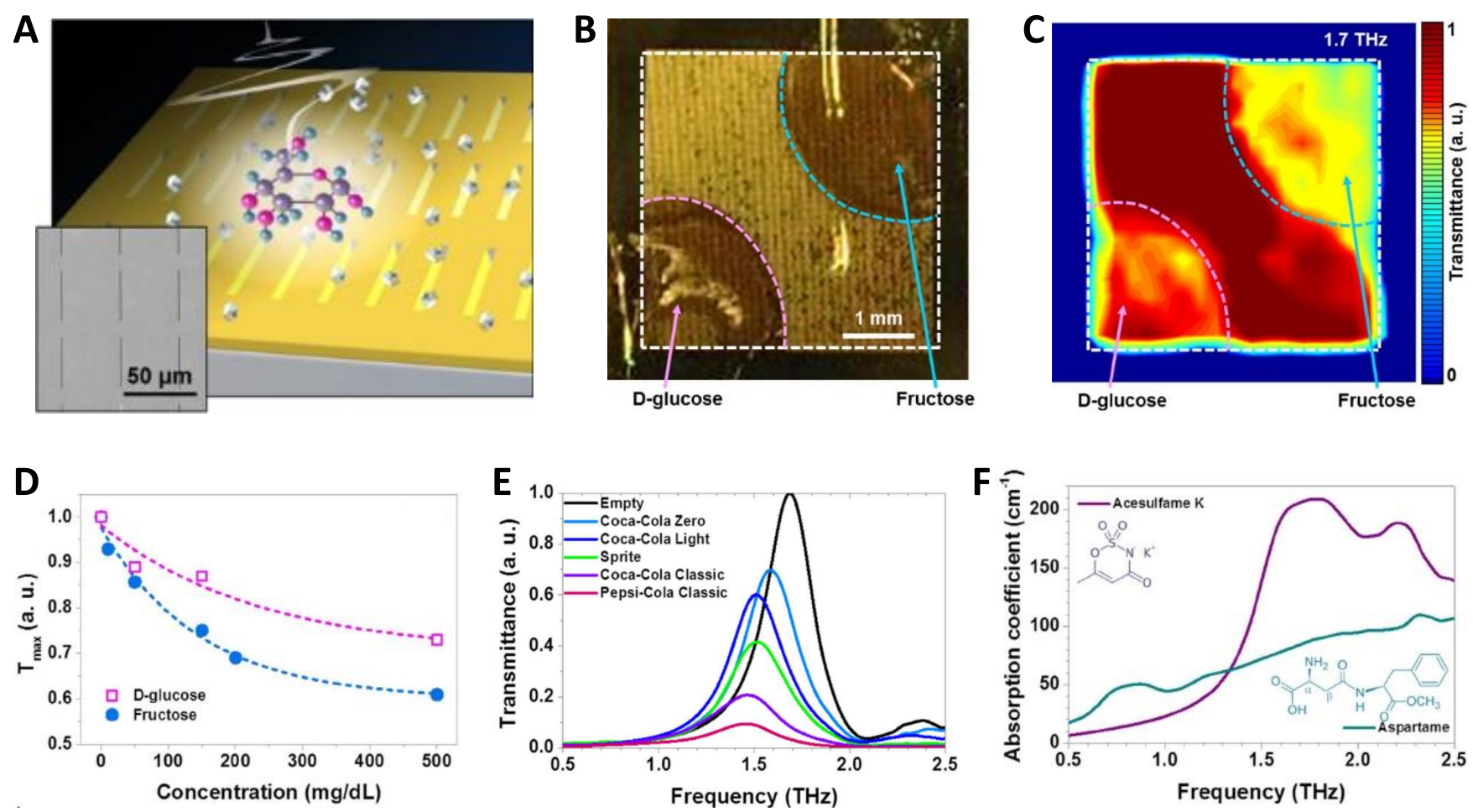

Figure 20. Highly-sensitive and selective nanoantenna. (A) Schematic of a THz nanoantenna for detection of sugar molecules. (B) Fructose antenna with stains of $250 \mathrm{mg} / \mathrm{dL}$ fructose (top-right) and D-glucose (bottom-left). (C) THz transmittance through stained fructose antenna illustrating the selective nature of nanoantenna. (D) THz transmittance peaks of fructose and D-glucose at different concentrations using fructose antenna. (E) THz transmittances of added sugars and artificial sweeteners in various branded beverages and dietary sodas using a fructose antenna. (F) THz absorption coefficients of low-concentrated artificial sweeteners: Acesulfame K and Aspartame. Reprinted with permission from Ref. ${ }^{88}$. (C) 2015, Springer Nature.

tenna, $\mathrm{THz}$ electromagnetic waves are made to be incident normally. As the $\mathrm{THz}$ waves pass through the dielectric film, the transmitted THz-electric field decays exponentially and is given by

$$
\frac{T_{S}(\omega)}{T_{r}(\omega)}=C e^{-\kappa k(\omega) h}
$$

here, $T_{S}=\left(E_{S}(\omega)\right)^{2}$ and $T_{r}(\omega)=\left(E_{r}\right)^{2}$ are the transmittances through dielectric and air nanogaps, respectively. In the above relation, $\mathrm{C}$ is known as the transmittance ratio at the air-dielectric interface, $k=\frac{2 \pi f}{c}$ is known as the incidence momentum and $\mathrm{h}$ is the thickness of the dielectric. Fig. $17(\mathrm{~B}-\mathrm{C})$ shows the simulated transmittance for different complex refractive indices. It is evident from the figure, that the absorption is dependent on the imaginary part of the complex refractive index $(\kappa)$, and the resonance frequency is solely dependent on the real part of the complex refractive index (n). However, the change in resonance frequency is quite small but stronger absorption can lead to an appreciable change. Therefore, the variation in the transmission spectra will provide evident proofs for identification of molecules and species contained in the biomaterial sample.

To study the effects of various gap-widths on THz- sensing, ${ }^{89}$ a single-slot $\mathrm{THz}$ nanoantennas of length 90 $\mu \mathrm{m}$ and slot-widths of $50 \mathrm{~nm}$ (50 nm thick), 100, 200, 500,1000 and $5000 \mathrm{~nm}$ (100 nm thick) was fabricated on a thick quartz substrate and filled with $1 \mathrm{mg} / \mathrm{ml} \mathrm{RDX}$ (1,3,5-trinitroperhydro-1,3,5-triazine) molecules. Initially, the THz-absorption $(\alpha)$ of $1 \mathrm{mg}$ RDX molecules placed over a bare quartz substrate was studied which is shown in Fig. 18 (B). Then the absorption of RDX molecules inside the various nano-slots was studied and the data obtained was plotted against the slot-widths as shown in the Fig. 18 (C). From Fig. 18 (B - C), it is evident that nanoantenna enhances the $\mathrm{THz}$-absorption by a large factor of $10^{3}$.

The effects of different substrates on biomaterial detection ${ }^{93}$ were studied using THz-antenna (length = $100 \mu \mathrm{m}$ ) with $10 \times 10$ slot-array each with a slot-width of $2 \mu \mathrm{m}$ and periodicity of $200 \mu \mathrm{m}$ (Fig. 19 (A - B)), was lithographed using electron-beam on two different substrates, Si (undoped) and quartz, to sense yeast samples. Using FFT (Fast Fourier Transform) algorithm, the normalized $\mathrm{THz}$-transmission amplitudes for $\mathrm{THz}$ slot-antenna, with (an average of 50 yeast molecules, or $\mathrm{N}_{a v}=50$ ) and without yeast sample was analyzed (Fig. 19 (C)). From the figure, an evident $9 \mathrm{GHz}$ redshift was observed in the resonance frequency. To com- 

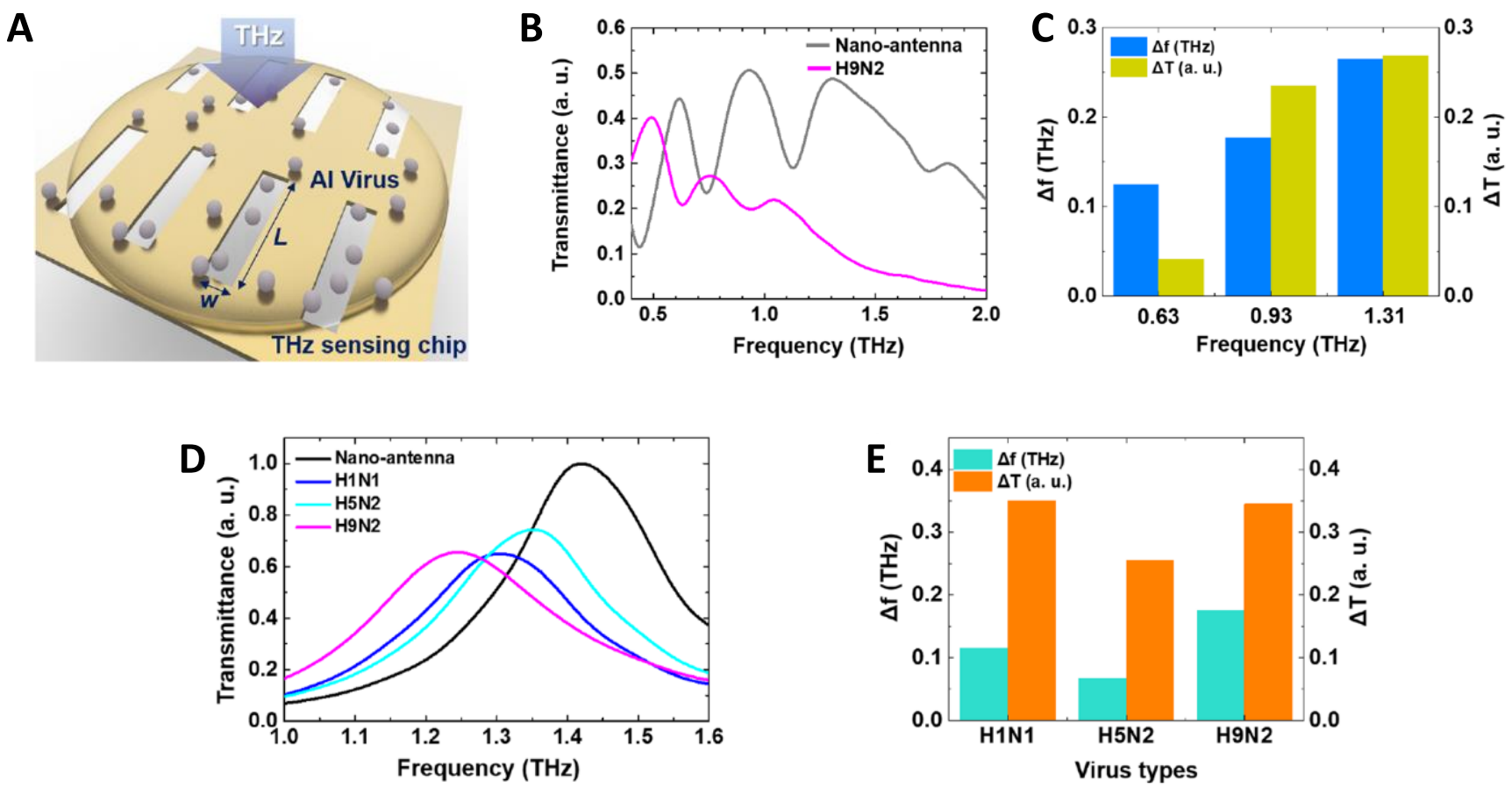

Figure 21. Detection of virus samples using THz nanoantenna sensing chip. (A) Schematic of a THz nano-slot antenna array sensing chip used to detect virus samples in liquid state. (B) Transmittance spectra measured using a multi-resonance nanoantenna, with and without H9N2. (C) Difference in transmitted intensity $(\Delta \mathrm{T})$ and resonance frequency shifts from each fundamental resonance peak $(\Delta \mathrm{f})$ for $\mathrm{H} 9 \mathrm{~N} 2$ virus sample. (D) THz transmission spectra through single-resonance nanoantenna, with and without virus samples (H1N1, H5N2 and H9N2). (E) Difference in transmitted intensity $(\Delta \mathrm{T})$ and resonance frequency shifts from the fundamental resonance peak $(\Delta \mathrm{f})$ of single-resonance nanoantenna for different virus samples. Reprinted with permission from Ref. ${ }^{92}$. (C) 2017, Springer Nature.

pare the effects of different substrate, the normalized THz-transmission amplitudes were measured for Siand quartz-substrate THz antennas as shown in Fig. 19 (D - E). Comparing the measured amplitudes, quartz showed stronger red-shifts compared to Si-substrate (Fig. $19(\mathrm{~F})$ ), showing $1 / \epsilon_{\text {eff }}$ dependence of the resonant frequency-shift $\left(\Delta \mathrm{f} / \mathrm{f}_{0}\right)$, where $\epsilon_{e f f}=n_{e f f}^{2}$ is known as effective dielectric constant. Hence it can be concluded that by using low dielectric constant substrate, the sensitivity of a THz nanoantenna can be enhanced.

$\mathrm{THz}$ nanoantennas are also selective in nature. ${ }^{88}$ This selective detection was shown using a nanoantenna of length $35 \mu \mathrm{m}$ having a resonance frequency of $1.7 \mathrm{THz}$. This antenna was specially designed to differentiate between fructose and D-glucose, and is also known as fructose antenna (Fig. 20 (B)). These molecules were discriminated from their measured transmittances using the fructose antenna as shown in Fig. 20 (C - D). Using the same nanoantenna, the sugars and low-concentrated artificial sweeteners (acesulfame $\mathrm{K}$ and aspartame) contained in beverages and dietary sodas, respectively, of popular brands were detected. The detection of these artificial sweeteners is important as these are recently found to be addictive and toxic in nature. The different transmittances of sugars contained in sweetened bever- ages of various brands in the $\mathrm{THz}$ range $(0.5-2.5 \mathrm{THz})$ are shown in Fig. 20 (E). The low transmittances seen in the figure are due to the two artificially added sweeteners whose THz-absorptions is shown in Fig. 20 (F). Hence, nanoantennas can provide highly sensitive detection even at low molecular concentration.

Recently, Lee et al..$^{92}$ studied the THz-transmittances of various virus samples (H1N1, H5N2, AND H9N2) using two THz nanoantennas: i) multi-resonance nanoantenna (with resonance frequencies of $0.63,0.93$ and 1.31 $\mathrm{THz}$ ), and ii) single-resonance nanoantenna (with resonance frequency of $1.4 \mathrm{THz}$ ), which are shown in Fig. 21 (B - E). Similar work has been reported by Park et al. ${ }^{94}$ in which they studied the transmission amplitude in both presence and absence of virus samples (PRD1 and MS2 viruses) using a THz nanogap metamaterial (Fig. 22).

\section{CONCLUSION AND FUTURE OUTLOOK}

$\mathrm{THz}$ waves are less penetrating and non-ionising electromagnetic waves. These waves are also known as 'sub-millimeter waves' and hence, is widely used in the fields of astronomy and spectroscopy. Since the description of electromagnetic interactions with metal is elucidated by using Maxwell's equations, researchers are 
A

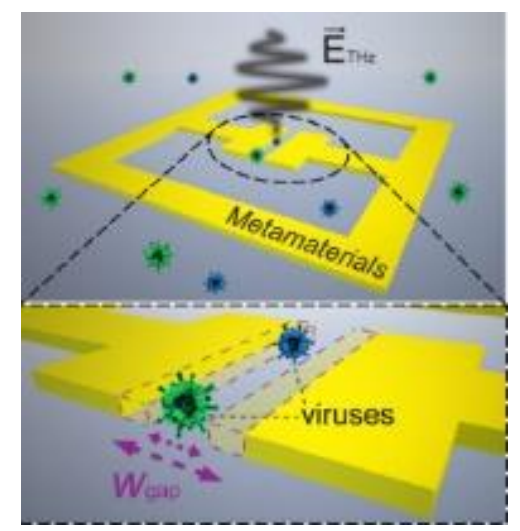

B

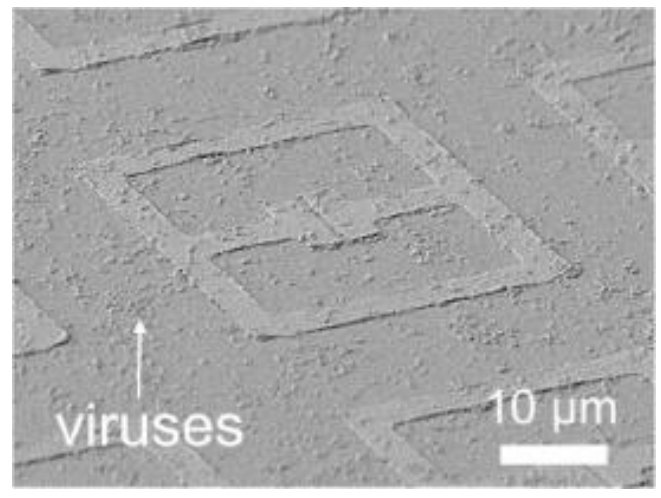

C

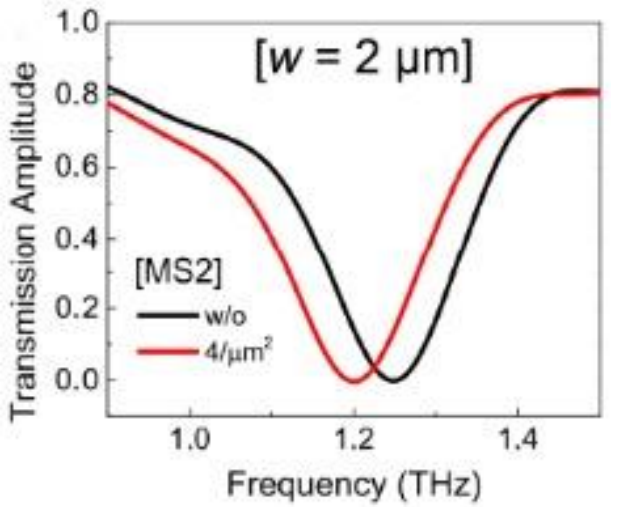

D

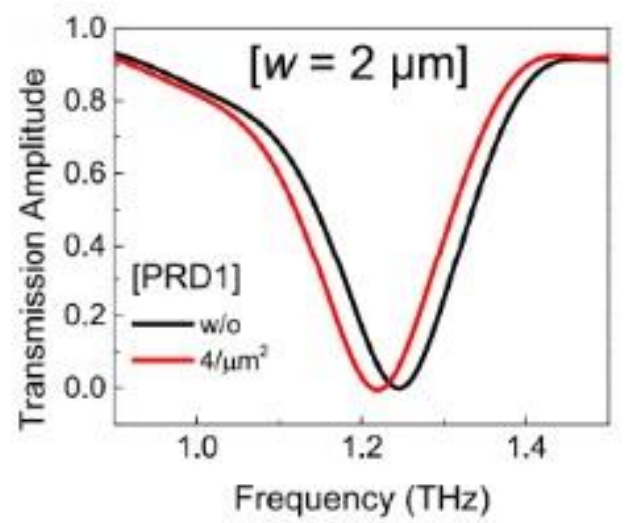

Figure 22. Detection of virus samples using $\mathrm{THz}$ nanogap metamaterial. (A) Schematic of $\mathrm{THz}$ nanogap metamaterial used to sense virus samples. (B) SEM image virus samples deposited on a THz nanogap metamaterial with $200 \mathrm{~nm}$ gap-width $(w)$. THz transmission spectra of THz metamaterial $(w=2 \mu m)$ containing (C) PRD1 and (D) MS2 virus samples. Noticeable red-shifts in resonance frequency are seen describing the sensitive detection of viruses using $\mathrm{THz}$ nanogap metamaterial. Reprinted with permission from Ref. ${ }^{94}$. ( 2017, Optical Society of America.

very much fascinated to study the electric field localization and other plasmonic effects in various metallic nanostructures. Numerous investigations reported that the electromagnetic interactions with metal film increase the mobility of charges in the film, tending it to move towards the metal edges, leading to the enhancement of the electric field inside different nanogap structures. To study the light-matter interactions, researchers are fascinated towards $\mathrm{THz}$ waves as they can squeeze through sub-nanometer metallic gaps (THz nanoantennas) and show non-linear optical responses. These nanoantennas are further integrated with different materials to study its novel plasmonic properties. Recently, successful fabrication of graphene-integrated plasmonic system $^{70,167,239-242}$ has boosted further investigations on their plasmonic properties because graphene is atomically thin and is electrically tunable.

As discussed earlier in this review, $\mathrm{THz}$ nanoantennas have played a crucial role as biosensors for ultrasensitive detection of various biomolecules and biomaterials, which is our prime focus in this review. We have come across various literatures in which we have seen the development of nanoantennas, becoming more sen- sitive and selective. Due to its enhanced detection in low molecular concentrations, in future nanoantennas can be used to monitor blood sugar level. ${ }^{88}$ As an emerging future technology, nanoantennas can be used for early detection of cancerous tumours ${ }^{243,244}$ which will lead to the development of effective cancer treatment. Few recent works have shown that biosensing based on surface plasmon resonance (SPR) has proved to be effective in label-free tumor detection ${ }^{245-248}$ and can be used to detect a single molecule of an early stage tumor. Currently, research and development are in progress in making a nanoantenna based SPR biosensor which will be able to detect cancer cells at an early stage.

\section{ACKNOWLEDGEMENT}

The authors thanks Birla Institute of Technology, Mesra, Ranchi for providing research facilities and MHRD, government of India for support through TEQIP - III. The authors also thanks Pawan Kumar Dubey, Akriti Raj, Sameer Kumar Tiwari, Kamana Mishra, Priyanshi Srivastava, Dhruv Sood and Devo- 
tosh Ganguly for useful discussions and proof reading of the manuscript.

VIII. KEYWORDS

\section{CONFLICT OF INTEREST}

The authors declares no conflict of interest.

* nara.laxmi@gmail.com

1 P. Bharadwaj, B. Deutsch, and L. Novotny, “Optical antennas," Advances in Optics and Photonics 1, 438 (2009).

2 P. Muhlschlegel, "Resonant optical antennas," Science 308, 1607 (2005).

3 J. Alda, J. M. Rico-García, J. M. López-Alonso, and G. Boreman, "Optical antennas for nano-photonic applications," Nanotechnology 16, S230 (2005).

4 B. M. Ross and L. P. Lee, "Comparison of near- and farfield measures for plasmon resonance of metallic nanoparticles," Optics Letters 34, 896 (2009).

5 A. Alù and N. Engheta, "Input impedance, nanocircuit loading, and radiation tuning of optical nanoantennas," Physical Review Letters 101 (2008), 10.1103/physrevlett.101.043901.

6 L. Novotny, "Effective wavelength scaling for optical antennas," Physical Review Letters 98 (2007), 10.1103/physrevlett.98.266802.

7 K. Kneipp, Y. Wang, H. Kneipp, L. T. Perelman, I. Itzkan, R. R. Dasari, and M. S. Feld, "Single molecule detection using surface-enhanced raman scattering (SERS)," Physical Review Letters 78, 1667 (1997).

8 S. Nie, "Probing single molecules and single nanoparticles by surface-enhanced raman scattering," Science 275, 1102 (1997).

9 H. Xu, E. J. Bjerneld, M. Kll, and L. Brjesson, "Spectroscopy of single hemoglobin molecules by surface enhanced raman scattering," Physical Review Letters 83, 4357 (1999).

10 M. L. Juan, M. Righini, and R. Quidant, "Plasmon nanooptical tweezers," Nature Photonics 5, 349 (2011).

11 O. M. Maragò, P. H. Jones, P. G. Gucciardi, G. Volpe, and A. C. Ferrari, "Optical trapping and manipulation of nanostructures," Nature Nanotechnology 8, 807 (2013).

12 D. R. Ward, F. Hser, F. Pauly, J. C. Cuevas, and D. Natelson, "Optical rectification and field enhancement in a plasmonic nanogap," Nature Nanotechnology 5, 732 (2010).

13 M. Kauranen and A. V. Zayats, "Nonlinear plasmonics," Nature Photonics 6, 737 (2012).

14 Y.-M. Bahk, G. Ramakrishnan, J. Choi, H. Song, G. Choi, Y. H. Kim, K. J. Ahn, D.-S. Kim, and P. C. M. Planken, "Plasmon enhanced terahertz emission from single layer graphene," ACS Nano 8, 9089 (2014).

15 J. B. Lassiter, X. Chen, X. Liu, C. Ciracì, T. B. Hoang, S. Larouche, S.-H. Oh, M. H. Mikkelsen, and D. R. Smith, "Third-harmonic generation enhancement by filmcoupled plasmonic stripe resonators," ACS Photonics 1, 1212 (2014).

16 Y.-M. Bahk, S. Han, J. Rhie, J. Park, H. Jeon, N. Park, and D.-S. Kim, "Ultimate terahertz field enhancement of single nanoslits," Physical Review B 95 (2017), 10.1103/phys- revb.95.075424.

17 Y.-M. Bahk, D.-S. Kim, and H.-R. Park, “Large-area metal gaps and their optical applications," Advanced Optical Materials 7, 1800426 (2018).

18 N. Bonod, E. Popov, D. Gérard, J. Wenger, and H. Rigneault, "Field enhancement in a circular aperture surrounded by a single channel groove," Optics Express 16, 2276 (2008).

19 H. Cao and A. Nahata, "Resonantly enhanced transmission of terahertz radiation through a periodic array of subwavelength apertures," Optics Express 12, 1004 (2004).

20 H.-T. Chen, W. J. Padilla, J. M. O. Zide, A. C. Gossard, A. J. Taylor, and R. D. Averitt, "Active terahertz metamaterial devices," Nature 444, 597 (2006).

21 X. Chen, H.-R. Park, N. C. Lindquist, J. Shaver, M. Pelton, and S.-H. Oh, "Squeezing millimeter waves through a single, nanometer-wide, centimeter-long slit," Scientific Reports 4 (2014), 10.1038/srep06722.

22 J. Jeong, J. Rhie, W. Jeon, C. S. Hwang, and D.-S. Kim, "High-throughput fabrication of infinitely long $10 \mathrm{~nm}$ slit arrays for terahertz applications," Journal of Infrared, Millimeter, and Terahertz Waves 36, 262 (2014).

23 J.-H. Kang, D.-S. Kim, and M. Seo, "Terahertz wave interaction with metallic nanostructures," Nanophotonics 7, 763 (2018).

24 J.-Y. Kim, B. J. Kang, J. Park, Y.-M. Bahk, W. T. Kim, J. Rhie, H. Jeon, F. Rotermund, and D.-S. Kim, “Terahertz quantum plasmonics of nanoslot antennas in nonlinear regime," Nano Letters 15, 6683 (2015).

25 N. Kim, S. In, D. Lee, J. Rhie, J. Jeong, D.-S. Kim, and N. Park, "Colossal terahertz field enhancement using splitring resonators with a sub-10 nm gap," ACS Photonics 5, 278 (2017).

26 J. W. Lee, M. A. Seo, D. H. Kang, K. S. Khim, S. C. Jeoung, and D. S. Kim, "Terahertz electromagnetic wave transmission through random arrays of single rectangular holes and slits in thin metallic sheets," Physical Review Letters 99 (2007), 10.1103/physrevlett.99.137401.

27 H. O. Moser, B. D. F. Casse, O. Wilhelmi, and B. T. Saw, "Terahertz response of a microfabricated rod-split-ringresonator electromagnetic metamaterial," Physical Review Letters 94 (2005), 10.1103/physrevlett.94.063901.

28 A. Novitsky, A. M. Ivinskaya, M. Zalkovskij, R. Malureanu, P. U. Jepsen, and A. V. Lavrinenko, "Non-resonant terahertz field enhancement in periodically arranged nanoslits," Journal of Applied Physics 112, 074318 (2012).

29 A. Novitsky, M. Zalkovskij, R. Malureanu, and A. Lavrinenko, "Microscopic model of the $\mathrm{THz}$ field enhancement in a metal nanoslit," Optics Communications 284, 5495 (2011). 
30 H. R. Park, Y. M. Park, H. S. Kim, J. S. Kyoung, M. A. Seo, D. J. Park, Y. H. Ahn, K. J. Ahn, and D. S. Kim, “Terahertz nanoresonators: Giant field enhancement and ultrabroadband performance," Applied Physics Letters 96, 121106 (2010).

31 D. Qu, D. Grischkowsky, and W. Zhang, “Terahertz transmission properties of thin, subwavelength metallic hole arrays," Optics Letters 29, 896 (2004).

32 J. G. Rivas, C. Schotsch, P. H. Bolivar, and H. Kurz, "Enhanced transmission of $\mathrm{THz}$ radiation through subwavelength holes," Physical Review B 68 (2003), 10.1103/physrevb.68.201306.

33 M. A. Seo, A. J. L. Adam, J. H. Kang, J. W. Lee, K. J. Ahn, Q. H. Park, P. C. M. Planken, and D. S. Kim, "Near field imaging of terahertz focusing onto rectangular apertures," Optics Express 16, 20484 (2008).

34 M. A. Seo, H. R. Park, S. M. Koo, D. J. Park, J. H. Kang, O. K. Suwal, S. S. Choi, P. C. M. Planken, G. S. Park, N. K. Park, Q. H. Park, and D. S. Kim, “Terahertz field enhancement by a metallic nano slit operating beyond the skindepth limit," Nature Photonics 3, 152 (2009).

35 M. Shalaby, H. Merbold, M. Peccianti, L. Razzari, G. Sharma, T. Ozaki, R. Morandotti, T. Feurer, A. Weber, L. Heyderman, B. Patterson, and H. Sigg, "Concurrent field enhancement and high transmission of $\mathrm{THz}$ radiation in nanoslit arrays," Applied Physics Letters 99, 041110 (2011).

36 A. Toma, S. Tuccio, M. Prato, F. D. Donato, A. Perucchi, P. D. Pietro, S. Marras, C. Liberale, R. P. Zaccaria, F. D. Angelis, L. Manna, S. Lupi, E. D. Fabrizio, and L. Razzari, "Squeezing terahertz light into nanovolumes: Nanoantenna enhanced terahertz spectroscopy (NETS) of semiconductor quantum dots," Nano Letters 15, 386 (2014).

37 L.-N. Tripathi, Y.-M. Bahk, G. Choi, S. Han, N. Park, and D.-S. Kim, "Terahertz transmission through rings of quantum dots-nanogap," Applied Physics Express 9, 032001 (2016).

38 C. A. Werley, K. Fan, A. C. Strikwerda, S. M. Teo, X. Zhang, R. D. Averitt, and K. A. Nelson, "Time-resolved imaging of near-fields in $\mathrm{THz}$ antennas and direct quantitative measurement of field enhancements," Optics Express 20, 8551 (2012).

39 W. Park, Y. Lee, T. Kang, J. Jeong, and D.-S. Kim, "Terahertz-driven polymerization of resists in nanoantennas," Scientific Reports 8 (2018), 10.1038/s41598-01826214-w.

40 H.-R. Park, Y.-M. Bahk, K. J. Ahn, Q.-H. Park, D.-S. Kim, L. Martín-Moreno, F. J. García-Vidal, and J. Bravo-Abad, "Controlling terahertz radiation with nanoscale metal barriers embedded in nano slot antennas," ACS Nano 5, 8340 (2011).

41 N. C. Lindquist, P. Nagpal, K. M. McPeak, D. J. Norris, and S.-H. Oh, "Engineering metallic nanostructures for plasmonics and nanophotonics," Reports on Progress in Physics 75, 036501 (2012).

42 M. C. Hoffmann, B. S. Monozon, D. Livshits, E. U. Rafailov, and D. Turchinovich, "Terahertz electro-absorption effect enabling femtosecond all-optical switching in semiconductor quantum dots," Applied Physics Letters 97, 231108 (2010).

43 H. Tanoto, J. H. Teng, Q. Y. Wu, M. Sun, Z. N. Chen, S. A. Maier, B. Wang, C. C. Chum, G. Y. Si, A. J. Danner, and S. J. Chua, "Greatly enhanced continuous-wave terahertz emission by nano-electrodes in a photoconductive photomixer," Nature Photonics 6, 121 (2012).

44 L. Ju, B. Geng, J. Horng, C. Girit, M. Martin, Z. Hao, H. A. Bechtel, X. Liang, A. Zettl, Y. R. Shen, and F. Wang, "Graphene plasmonics for tunable terahertz metamaterials," Nature Nanotechnology 6, 630 (2011).

45 M. Shur, "Plasma wave terahertz electronics," Electronics Letters 46, S18 (2010).

46 P. Upadhyaya, S. Pramanik, and S. Bandyopadhyay, "Optical transitions in a quantum wire with spin-orbit interaction and its applications in terahertz electronics: Beyond zeroth-order theory," Physical Review B 77 (2008), 10.1103/physrevb.77.155439.

47 M. Bahramipanah, M. S. Abrishamian, S. A. Mirtaheri, and J.-M. Liu, "Ultracompact plasmonic loop-stub notch filter and sensor," Sensors and Actuators B: Chemical 194, 311 (2014).

48 D. M. Mittleman, "Frontiers in terahertz sources and plasmonics," Nature Photonics 7, 666 (2013).

49 C. R. Williams, S. R. Andrews, S. A. Maier, A. I. FernándezDomínguez, L. Martín-Moreno, and F. J. García-Vidal, "Highly confined guiding of terahertz surface plasmon polaritons on structured metal surfaces," Nature Photonics 2, 175 (2008).

50 A. J. Fitzgerald, E. Berry, N. N. Zinovev, G. C. Walker, M. A. Smith, and J. M. Chamberlain, "An introduction to medical imaging with coherent terahertz frequency radiation," Physics in Medicine and Biology 47, R67 (2002).

51 E. P. J. Parrott, Y. Sun, and E. Pickwell-MacPherson, "Terahertz spectroscopy: Its future role in medical diagnoses," Journal of Molecular Structure 1006, 66 (2011).

52 J. W. Handley, A. J. Fitzgerald, E. Berry, and R. D. Boyle, "Wavelet compression in medical terahertz pulsed imaging," Physics in Medicine and Biology 47, 3885 (2002).

53 W. Tu, S. Zhong, Y. Shen, and A. Incecik, "Nondestructive testing of marine protective coatings using terahertz waves with stationary wavelet transform," Ocean Engineering 111, 582 (2016).

$54 \mathrm{~J}$. Li and Y. Pi, "Target detection for terahertz radar networks based on micro-doppler signatures," International Journal of Sensor Networks 17, 115 (2015).

55 E. N. Semashkin and T. V. Artyushkina, "Operating range and all-weather capability of terahertz $(01 \mathrm{THz})$ and gigahertz (3-333 GHz) radars on horizontal and oblique tracks," Journal of Optical Technology 82, 430 (2015).

56 D. Zimdars, J. S. White, G. Stuk, A. Chernovsky, G. Fichter, and S. Williamson, "Security and non destructive evaluation application of high speed time domain terahertz imaging," in 2006 Conference on Lasers and Electro-Optics and 2006 Quantum Electronics and Laser Science Conference (IEEE, 2006).

57 S.-P. Han, N. Kim, W.-H. Lee, E. S. Lee, H. Ko, I.-M. Lee, K. Moon, D. H. Lee, and K. H. Park, "Real-time imaging of moving living objects using a compact terahertz scanner," Applied Physics Express 9, 022501 (2016).

58 K. Fukunaga and I. Hosako, "Innovative non-invasive analysis techniques for cultural heritage using terahertz technology," Comptes Rendus Physique 11, 519 (2010).

59 J.-M. Manceau, A. Nevin, C. Fotakis, and S. Tzortzakis, "Terahertz time domain spectroscopy for the analysis of cultural heritage related materials," Applied Physics B 90, 365 (2008). 
60 J. W. Lee, M. A. Seo, D. J. Park, S. C. Jeoung, Q. H. Park, C. Lienau, and D. S. Kim, "Terahertz transparency at fabry-perot resonances of periodic slit arrays in a metal plate: experiment and theory," Optics Express 14, 12637 (2006).

61 I. H. Libon, S. Baumgrtner, M. Hempel, N. E. Hecker, J. Feldmann, M. Koch, and P. Dawson, "An optically controllable terahertz filter," Applied Physics Letters 76, 2821 (2000).

62 R. Mendis, A. Nag, F. Chen, and D. M. Mittleman, "A tunable universal terahertz filter using artificial dielectrics based on parallel-plate waveguides," Applied Physics Letters 97, 131106 (2010).

63 M. A. Kaliteevski, S. Brand, J. Garvie-Cook, R. A. Abram, and J. M. Chamberlain, "Terahertz filter based on refractive properties of metallic photonic crystal," Optics Express 16, 7330 (2008).

64 F. Lan, Z. Yang, L. Qi, X. Gao, and Z. Shi, “Terahertz dual-resonance bandpass filter using bilayer reformative complementary metamaterial structures," Optics Letters 39, 1709 (2014).

65 B. Heshmat, H. Pahlevaninezhad, P. Yuanjie, M. MasnadiShirazi, R. B. Lewis, T. Tiedje, R. Gordon, and T. E. Darcie, "Nanoplasmonic terahertz photoconductive switch on GaAs," Nano Letters 12, 6255 (2012).

66 M. Rahm, J.-S. Li, and W. J. Padilla, "THz wave modulators: A brief review on different modulation techniques," Journal of Infrared, Millimeter, and Terahertz Waves 34, 1 (2012).

67 S. B. Choi, J. S. Kyoung, H. S. Kim, H. R. Park, D. J. Park, B.-J. Kim, Y. H. Ahn, F. Rotermund, H.-T. Kim, K. J. Ahn, and D. S. Kim, "Nanopattern enabled terahertz alloptical switching on vanadium dioxide thin film," Applied Physics Letters 98, 071105 (2011).

68 M. Unlu and M. Jarrahi, "Miniature multi-contact MEMS switch for broadband terahertz modulation," Optics Express 22, 32245 (2014).

69 J. Li, J. He, and Z. Hong, “Terahertz wave switch based on silicon photonic crystals," Applied Optics 46, 5034 (2007).

70 W. Gao, J. Shu, K. Reichel, D. V. Nickel, X. He, G. Shi, R. Vajtai, P. M. Ajayan, J. Kono, D. M. Mittleman, and Q. Xu, "High-contrast terahertz wave modulation by gated graphene enhanced by extraordinary transmission through ring apertures," Nano Letters 14, 1242 (2014).

71 G. Liu, M. He, Z. Tian, J. Li, and J. Liu, "Terahertz surface plasmon sensor for distinguishing gasolines," Applied Optics 52, 5695 (2013).

72 V. Astley, K. Reichel, R. Mendis, and D. M. Mittleman, "Terahertz microfluidic sensing using a parallel-plate waveguide sensor," Journal of Visualized Experiments (2012), 10.3791/4304.

73 F. Alves, D. Grbovic, B. Kearney, and G. Karunasiri, "Microelectromechanical systems bimaterial terahertz sensor with integrated metamaterial absorber," Optics Letters 37, 1886 (2012).

74 A. Hassani and M. Skorobogatiy, "Surface plasmon resonance-like integrated sensor at terahertz frequencies for gaseous analytes," Optics Express 16, 20206 (2008).

75 F. Miyamaru, S. Hayashi, C. Otani, K. Kawase, Y. Ogawa, H. Yoshida, and E. Kato, "Terahertz surface-wave resonant sensor with a metal hole array," Optics Letters 31, 1118 (2006).
${ }^{76} \mathrm{~K} . \mathrm{Xu}$, "Integrated silicon directly modulated light source using p-well in standard CMOS technology," IEEE Sensors Journal 16, 6184 (2016).

77 Y.-S. Lee, Principles of Terahertz Science and Technology (SPRINGER NATURE, 2008).

78 J. B. Baxter and G. W. Guglietta, "Terahertz spectroscopy," Analytical Chemistry 83, 4342 (2011).

79 J. X. Xi-Cheng Zhang, Introduction to THz Wave Photonics (Springer-Verlag GmbH, 2009).

80 S. Dexheimer, ed., Terahertz Spectroscopy (CRC Press, 2007).

81 M. Nagel, M. Frst, and H. Kurz, "THz biosensing devices: fundamentals and technology," Journal of Physics: Condensed Matter 18, S601 (2006).

82 S. Kar, V. L. Nguyen, D. R. Mohapatra, Y. H. Lee, and A. K. Sood, "Ultrafast spectral photoresponse of bilayer graphene: Optical pump-terahertz probe spectroscopy," ACS Nano 12, 1785 (2018).

83 B. M. Fischer, M. Walther, and P. U. Jepsen, "Far-infrared vibrational modes of DNA components studied by terahertz time-domain spectroscopy," Physics in Medicine and Biology 47, 3807 (2002).

84 A. Markelz, S. Whitmire, J. Hillebrecht, and R. Birge, “THz time domain spectroscopy of biomolecular conformational modes," Physics in Medicine and Biology 47, 3797 (2002).

85 A. Arora, T. Q. Luong, M. Krger, Y. J. Kim, C.-H. Nam, A. Manz, and M. Havenith, "Terahertz-time domain spectroscopy for the detection of PCR amplified DNA in aqueous solution," The Analyst 137, 575 (2012).

86 X. Yang, D. Wei, S. Yan, Y. Liu, S. Yu, M. Zhang, Z. Yang, X. Zhu, Q. Huang, H.-L. Cui, and W. Fu, "Rapid and label-free detection and assessment of bacteria by terahertz time-domain spectroscopy," Journal of Biophotonics 9, 1050 (2016).

87 X. Yang, K. Yang, Y. Luo, and W. Fu, "Terahertz spectroscopy for bacterial detection: opportunities and challenges," Applied Microbiology and Biotechnology 100, 5289 (2016).

88 D.-K. Lee, J.-H. Kang, J.-S. Lee, H.-S. Kim, C. Kim, J. H. Kim, T. Lee, J.-H. Son, Q.-H. Park, and M. Seo, "Highly sensitive and selective sugar detection by terahertz nanoantennas," Scientific Reports 5 (2015), 10.1038/srep15459.

89 H.-R. Park, K. J. Ahn, S. Han, Y.-M. Bahk, N. Park, and D.-S. Kim, "Colossal absorption of molecules inside single terahertz nanoantennas," Nano Letters 13, 1782 (2013).

90 O. Limaj, D. Etezadi, N. J. Wittenberg, D. Rodrigo, D. Yoo, S.-H. Oh, and H. Altug, "Infrared plasmonic biosensor for real-time and label-free monitoring of lipid membranes," Nano Letters 16, 1502 (2016).

91 D. Rodrigo, O. Limaj, D. Janner, D. Etezadi, F. J. G. de Abajo, V. Pruneri, and H. Altug, "Mid-infrared plasmonic biosensing with graphene," Science 349, 165 (2015).

92 D.-K. Lee, J.-H. Kang, J. Kwon, J.-S. Lee, S. Lee, D. H. Woo, J. H. Kim, C.-S. Song, Q.-H. Park, and M. Seo, "Nano metamaterials for ultrasensitive terahertz biosensing," Scientific Reports 7 (2017), 10.1038/s41598-017-08508-7.

93 S. J. Park, J. T. Hong, S. J. Choi, H. S. Kim, W. K. Park, S. T. Han, J. Y. Park, S. Lee, D. S. Kim, and Y. H. Ahn, "Detection of microorganisms using terahertz metamaterials," Scientific Reports 4 (2014), 10.1038/srep04988.

94 S. J. Park, S. H. Cha, G. A. Shin, and Y. H. Ahn, "Sensing viruses using terahertz nano-gap metamaterials," Biomedical Optics Express 8, 3551 (2017). 
95 X. Chen, H.-R. Park, M. Pelton, X. Piao, N. C. Lindquist, H. Im, Y. J. Kim, J. S. Ahn, K. J. Ahn, N. Park, D.-S. Kim, and S.-H. Oh, "Atomic layer lithography of waferscale nanogap arrays for extreme confinement of electromagnetic waves," Nature Communications 4 (2013), $10.1038 /$ ncomms3361.

96 J. Lee, M. Seo, D. Park, D. Kim, S. Jeoung, C. Lienau, Q.-H. Park, and P. Planken, "Shape resonance omni-directional terahertz filters with near-unity transmittance," Optics Express 14, 1253 (2006).

97 J. W. Lee, M. A. Seo, D. S. Kim, S. C. Jeoung, C. Lienau, J. H. Kang, and Q.-H. Park, "Fabry-perot effects in THz timedomain spectroscopy of plasmonic band-gap structures," Applied Physics Letters 88, 071114 (2006).

98 D. Ward, E. Statz, and K. Nelson, "Fabrication of polaritonic structures in $\mathrm{LiNbO} 3$ and $\mathrm{LiTaO} 3$ using femtosecond laser machining," Applied Physics A 86, 49 (2006).

99 C. Enkrich, F. Pérez-Willard, D. Gerthsen, J. F. Zhou, T. Koschny, C. M. Soukoulis, M. Wegener, and S. Linden, "Focused-ion-beam nanofabrication of near-infrared magnetic metamaterials," Advanced Materials 17, 2547 (2005).

100 N. Ocelic and R. Hillenbrand, "Subwavelength-scale tailoring of surface phonon polaritons by focused ion-beam implantation," Nature Materials 3, 606 (2004).

101 H. F. Ghaemi, T. Thio, D. E. Grupp, T. W. Ebbesen, and H. J. Lezec, "Surface plasmons enhance optical transmission through subwavelength holes," Physical Review B 58, 6779 (1998).

102 T. W. Ebbesen, H. J. Lezec, H. F. Ghaemi, T. Thio, and P. A. Wolff, "Extraordinary optical transmission through sub-wavelength hole arrays," Nature 391, 667 (1998).

103 H.-R. Park, Y.-M. Bahk, J. H. Choe, S. Han, S. S. Choi, K. J. Ahn, N. Park, Q.-H. Park, and D.-S. Kim, "Terahertz pinch harmonics enabled by single nano rods," Optics Express 19, 24775 (2011).

104 D. C. Bell, M. C. Lemme, L. A. Stern, J. R. Williams, and C. M. Marcus, "Precision cutting and patterning of graphene with helium ions," Nanotechnology 20, 455301 (2009).

105 H.-T. Chen, J. F. O'Hara, A. K. Azad, A. J. Taylor, R. D. Averitt, D. B. Shrekenhamer, and W. J. Padilla, "Experimental demonstration of frequency-agile terahertz metamaterials," Nature Photonics 2, 295 (2008).

106 R. Singh, E. Plum, C. Menzel, C. Rockstuhl, A. K. Azad, R. A. Cheville, F. Lederer, W. Zhang, and N. I. Zheludev, "Terahertz metamaterial with asymmetric transmission," Physical Review B 80 (2009), 10.1103/physrevb.80.153104.

107 M. Choi, S. H. Lee, Y. Kim, S. B. Kang, J. Shin, M. H. Kwak, K.-Y. Kang, Y.-H. Lee, N. Park, and B. Min, "A terahertz metamaterial with unnaturally high refractive index," Nature 470, 369 (2011).

108 C. G. Willson, R. R. Dammel, and A. Reiser, "Photoresist materials: a historical perspective," in Metrology, Inspection, and Process Control for Microlithography XI (SPIE, 1997).

109 C. Lin, C. Chen, G. J. Schneider, P. Yao, S. Shi, A. Sharkawy, and D. W. Prather, "Wavelength scale terahertz twodimensional photonic crystal waveguides," Optics Express 12, 5723 (2004).

110 H.-R. Park, X. Chen, N.-C. Nguyen, J. Peraire, and S.-H. Oh, "Nanogap-enhanced terahertz sensing of $1 \mathrm{~nm}$ thick $(\lambda / 106)$ dielectric films," ACS Photonics 2, 417 (2015).

111 A. Fursina, S. Lee, R. G. S. Sofin, I. V. Shvets, and D. Natelson, "Nanogaps with very large aspect ratios for electrical measurements," Applied Physics Letters 92, 113102 (2008).

112 C. Vieu, F. Carcenac, A. Pépin, Y. Chen, M. Mejias, A. Lebib, L. Manin-Ferlazzo, L. Couraud, and H. Launois, "Electron beam lithography: resolution limits and applications," Applied Surface Science 164, 111 (2000).

113 A. Broers, A. Hoole, and J. Ryan, "Electron beam lithography-resolution limits," Microelectronic Engineering 32, 131 (1996).

114 Y. Chen, "Nanofabrication by electron beam lithography and its applications: A review," Microelectronic Engineering 135, 57 (2015).

115 A. Tseng, K. Chen, C. Chen, and K. Ma, "Electron beam lithography in nanoscale fabrication: recent development," IEEE Transactions on Electronics Packaging Manufacturing 26, 141 (2003).

116 J. C. Hulteen and R. P. V. Duyne, "Nanosphere lithography: A materials general fabrication process for periodic particle array surfaces," Journal of Vacuum Science \& Technology A: Vacuum, Surfaces, and Films 13, 1553 (1995).

117 P. Vettiger, "Nanometer sidewall lithography by resist silylation," Journal of Vacuum Science \& Technology B: Microelectronics and Nanometer Structures 7, 1756 (1989).

118 S. Y. Chou, P. R. Krauss, and P. J. Renstrom, "Imprint lithography with 25-nanometer resolution," Science 272, 85 (1996).

119 S. Y. Chou, "Nanoimprint lithography," Journal of Vacuum Science \& Technology B: Microelectronics and Nanometer Structures 14, 4129 (1996).

120 M. D. Austin, H. Ge, W. Wu, M. Li, Z. Yu, D. Wasserman, S. A. Lyon, and S. Y. Chou, "Fabrication of 5nm linewidth and 14nm pitch features by nanoimprint lithography," Applied Physics Letters 84, 5299 (2004).

121 L. Guo, “Nanoimprint lithography: Methods and material requirements," Advanced Materials 19, 495 (2007).

122 L. Guo, P. R. Krauss, and S. Y. Chou, "Nanoscale silicon field effect transistors fabricated using imprint lithography," Applied Physics Letters 71, 1881 (1997).

123 S. C. Ambhire, S. Palkhivala, A. Agrawal, A. Gupta, G. Rana, R. Mehta, D. Ghindani, A. Bhattacharya, V. G. Achanta, and S. S. Prabhu, "'pattern and peel" method for fabricating mechanically tunable terahertz metasurface on an elastomeric substrate," Optical Materials Express 8, 3382 (2018).

124 C. Feuillet-Palma, Y. Todorov, A. Vasanelli, and C. Sirtori, "Strong near field enhancement in THz nano-antenna arrays," Scientific Reports 3 (2013), 10.1038/srep01361.

125 O. K. Suwal, J. Rhie, N. Kim, and D.-S. Kim, "Nonresonant 104 terahertz field enhancement with 5-nm slits," Scientific Reports 7 (2017), 10.1038/srep45638.

126 W. Park, J. Rhie, N. Y. Kim, S. Hong, and D.-S. Kim, “Sub$10 \mathrm{~nm}$ feature chromium photomasks for contact lithography patterning of square metal ring arrays," Scientific Reports 6 (2016), 10.1038/srep23823.

127 H. Im, K. C. Bantz, N. C. Lindquist, C. L. Haynes, and S.H. Oh, "Vertically oriented sub-10-nm plasmonic nanogap arrays," Nano Letters 10, 2231 (2010).

128 H. T. Miyazaki and Y. Kurokawa, "Squeezing visible light waves into a 3-nm-thick and 55-nm-long plasmon cavity," Physical Review Letters 96 (2006), 10.1103/physrevlett.96.097401.

129 W. Zhu, M. G. Banaee, D. Wang, Y. Chu, and K. B. Crozier, "Lithographically fabricated optical antennas with gaps 
well below $10 \mathrm{~nm}, "$ Small 7, 1761 (2011).

130 W. Fan, S. Zhang, K. J. Malloy, and S. R. J. Brueck, "Enhanced mid-infrared transmission through nanoscale metallic coaxial-aperture arrays," Optics Express 13, 4406 (2005).

131 D. Ji, A. Cheney, N. Zhang, H. Song, J. Gao, X. Zeng, H. Hu, S. Jiang, Z. Yu, and Q. Gan, "Efficient midinfrared light confinement within sub-5-nm gaps for extreme field enhancement," Advanced Optical Materials 5, 1700223 (2017).

132 T. Ding, D. Sigle, L. Zhang, J. Mertens, B. de Nijs, and J. Baumberg, "Controllable tuning plasmonic coupling with nanoscale oxidation," ACS Nano 9, 6110 (2015).

133 L. N. Tripathi, T. Kang, Y.-M. Bahk, S. Han, G. Choi, J. Rhie, J. Jeong, and D.-S. Kim, "Quantum dots-nanogap metamaterials fabrication by self-assembly lithography and photoluminescence studies," Optics Express 23, 14937 (2015).

134 J. H. Kang, J.-H. Choe, D. S. Kim, and Q.-H. Park, “Substrate effect on aperture resonances in a thin metal film," Optics Express 17, 15652 (2009).

135 J.-H. Choe, J.-H. Kang, D.-S. Kim, and Q.-H. Park, “Slot antenna as a bound charge oscillator," Optics Express 20, 6521 (2012).

136 F. J. García-Vidal, L. Martín-Moreno, E. Moreno, L. K. S. Kumar, and R. Gordon, "Transmission of light through a single rectangular hole in a real metal," Physical Review B 74 (2006), 10.1103/physrevb.74.153411.

137 S.-G. Park, Y. Choi, Y.-J. Oh, and K.-H. Jeong, "Terahertz photoconductive antenna with metal nanoislands," Optics Express 20, 25530 (2012).

138 Y. Yang, R. Singh, and W. Zhang, "Anomalous terahertz transmission in bow-tie plasmonic antenna apertures," Optics Letters 36, 2901 (2011).

139 I. S. Maksymov, A. E. Miroshnichenko, and Y. S. Kivshar, "Actively tunable bistable optical yagi-uda nanoantenna," Optics Express 20, 8929 (2012).

140 T. H. Taminiau, F. D. Stefani, and N. F. van Hulst, "Enhanced directional excitation and emission of single emitters by a nano-optical yagi-uda antenna," Optics Express 16, 10858 (2008).

141 G. Pelosi and S. Selleri, "Babinet's principle in electromagnetics: Why does a slot radiate like a dipole? [historical corner]," IEEE Antennas and Propagation Magazine 59, 144 (2017).

142 H. Booker, "Slot aerials and their relation to complementary wire aerials (babinet's principle)," Journal of the Institution of Electrical Engineers - Part IIIA: Radiolocation 93, 620 (1946).

143 Y. Mushiake, “A report on japanese development of antennas: from the yagi-uda antenna to self-complementary antennas," IEEE Antennas and Propagation Magazine 46, 47 (2004).

144 M. Born and E. Wolf, Principles of Optics: Electromagnetic Theory of Propagation Interference and Diffraction of Light (Pergamon Pr, 1981).

145 S. Koo, M. S. Kumar, J. Shin, D. Kim, and N. Park, "Extraordinary magnetic field enhancement with metallic nanowire: Role of surface impedance in babinet's principle for sub-skin-depth regime," Physical Review Letters 103 (2009), 10.1103/physrevlett.103.263901.

146 L. Razzari, A. Toma, M. Clerici, M. Shalaby, G. Das, C. Liberale, M. Chirumamilla, R. P. Zaccaria, F. D. Angelis, M. Peccianti, R. Morandotti, and E. D. Fabrizio, "Tera- hertz dipole nanoantenna arrays: Resonance characteristics," Plasmonics 8, 133 (2012).

147 Y.-G. Jeong, M. J. Paul, S.-H. Kim, K.-J. Yee, D.-S. Kim, and Y.-S. Lee, "Large enhancement of nonlinear terahertz absorption in intrinsic GaAs by plasmonic nano antennas," Applied Physics Letters 103, 171109 (2013).

148 J. Shu, C. Qiu, V. Astley, D. Nickel, D. M. Mittleman, and Q. Xu, "High-contrast terahertz modulator based on extraordinary transmission through a ring aperture," Optics Express 19, 26666 (2011).

149 H. Merbold, A. Bitzer, and T. Feurer, "Second harmonic generation based on strong field enhancement in nanostructured THz materials," Optics Express 19, 7262 (2011).

150 L. Dai and C. Jiang, "Anomalous near-perfect extraordinary optical absorption on subwavelength thin metal film grating.," Optics Express 17, 20502 (2009).

151 B. gt, R. Vogelgesang, W. Sigle, N. Talebi, C. T. Koch, and P. A. van Aken, "Hybridized metal slit eigenmodes as an illustration of babinet's principle," ACS Nano 5, 6701 (2011).

152 J. Yang, Q. Cao, and C. Zhou, “Theory for terahertz plasmons of metallic nanowires with sub-skin-depth diameters," Optics Express 18, 18550 (2010).

153 D. Hu, X. Wang, S. Feng, J. Ye, W. Sun, Q. Kan, P. J. Klar, and Y. Zhang, "Ultrathin terahertz planar elements," Advanced Optical Materials 1, 186 (2013).

154 K. Iwaszczuk, A. Andryieuski, A. Lavrinenko, X.-C. Zhang, and P. U. Jepsen, "Terahertz field enhancement to the MV / cm regime in a tapered parallel plate waveguide," Optics Express 20, 8344 (2012).

155 D. S. Bulgarevich, M. Watanabe, and M. Shiwa, "Single sub-wavelength aperture with greatly enhanced transmission," New Journal of Physics 14, 053001 (2012).

156 M. N. Gadalla, M. Abdel-Rahman, and A. Shamim, “Design, optimization and fabrication of a $28.3 \mathrm{THz}$ nanorectenna for infrared detection and rectification," Scientific Reports 4 (2014), 10.1038/srep04270.

157 F. Fan, S.-T. Xu, X.-H. Wang, and S.-J. Chang, "Terahertz polarization converter and one-way transmission based on double-layer magneto-plasmonics of magnetized InSb," Optics Express 24, 26431 (2016).

158 T. Low and P. Avouris, "Graphene plasmonics for terahertz to mid-infrared applications," ACS Nano 8, 1086 (2014).

159 F. Fan, W.-H. Gu, S. Chen, X.-H. Wang, and S.-J. Chang, "State conversion based on terahertz plasmonics with vanadium dioxide coating controlled by optical pumping," Optics Letters 38, 1582 (2013).

160 A. H. Panaretos and D. H. Werner, "Spoof plasmon radiation using sinusoidally modulated corrugated reactance surfaces," Optics Express 24, 2443 (2016).

161 N. Yu, Q. J. Wang, M. A. Kats, J. A. Fan, S. P. Khanna, L. Li, A. G. Davies, E. H. Linfield, and F. Capasso, "Designer spoof surface plasmon structures collimate terahertz laser beams," Nature Materials 9, 730 (2010).

162 A. Ishikawa, S. Zhang, D. A. Genov, G. Bartal, and $X$. Zhang, "Deep subwavelength terahertz waveguides using gap magnetic plasmon," Physical Review Letters 102 (2009), 10.1103/physrevlett.102.043904.

163 R.-L. Chern, "Magnetic and surface plasmon resonances for periodic lattices of plasmonic split-ring resonators," Physical Review B 78 (2008), 10.1103/physrevb.78.085116.

164 D. Martin-Cano, O. Quevedo-Teruel, E. Moreno, L. MartinMoreno, and F. J. Garcia-Vidal, “Waveguided spoof sur- 
face plasmons with deep-subwavelength lateral confinement," Optics Letters 36, 4635 (2011).

165 S. H. Lee, J. Choi, H.-D. Kim, H. Choi, and B. Min, “Ultrafast refractive index control of a terahertz graphene metamaterial," Scientific Reports 3 (2013), 10.1038/srep02135.

166 D. Wang, Y. Gu, Y. Gong, C.-W. Qiu, and M. Hong, “An ultrathin terahertz quarter-wave plate using planar babinetinverted metasurface," Optics Express 23, 11114 (2015).

167 S.-F. Shi, B. Zeng, H.-L. Han, X. Hong, H.-Z. Tsai, H. S. Jung, A. Zettl, M. F. Crommie, and F. Wang, "Optimizing broadband terahertz modulation with hybrid graphene/metasurface structures," Nano Letters 15, 372 (2014).

168 K. Takano, K. Shibuya, K. Akiyama, T. Nagashima, F. Miyamaru, and M. Hangyo, "A metal-to-insulator transition in cut-wire-grid metamaterials in the terahertz region," Journal of Applied Physics 107, 024907 (2010).

169 A. Sommerfeld, "Mathematical theory of diffraction," in Mathematical Theory of Diffraction (Birkhuser Boston, 2004) pp. 9-68.

170 C. J. R. Sheppard, J. Lin, and S. S. Kou, "Rayleigh-sommerfeld diffraction formula in k space," Journal of the Optical Society of America A 30, 1180 (2013).

171 A. S. Marathay and J. F. McCalmont, "On the usual approximation used in the rayleigh-sommerfeld diffraction theory," Journal of the Optical Society of America A 21, 510 (2004).

172 H. A. Bethe, "Theory of diffraction by small holes," Physical Review 66, 163 (1944).

173 C. Bouwkamp and H. Casimir, "On multipole expansions in the theory of electromagnetic radiation," Physica 20, 539 (1954).

174 C. J. Bouwkamp, "Diffraction theory," Reports on Progress in Physics 17, 35 (1954).

175 R. Ulrich, "Far-infrared properties of metallic mesh and its complementary structure," Infrared Physics 7, 37 (1967).

176 T. J. Kim, T. Thio, T. W. Ebbesen, D. E. Grupp, and H. J. Lezec, "Control of optical transmission through metals perforated with subwavelength hole arrays," Optics Letters 24, 256 (1999).

177 D. S. Kim, S. C. Hohng, V. Malyarchuk, Y. C. Yoon, Y. H. Ahn, K. J. Yee, J. W. Park, J. Kim, Q. H. Park, and C. Lienau, "Microscopic origin of surface-plasmon radiation in plasmonic band-gap nanostructures," Physical Review Letters 91 (2003), 10.1103/physrevlett.91.143901.

178 B. K. Minhas, W. Fan, K. Agi, S. R. J. Brueck, and K. J. Malloy, "Metallic inductive and capacitive grids: theory and experiment," Journal of the Optical Society of America A 19, 1352 (2002).

179 A. Naweed, F. Baumann, J. William A. Bailey, A. S. Karakashian, and W. D. Goodhue, "Evidence for radiative damping in surface-plasmon-mediated light transmission through perforated conducting films," Journal of the Optical Society of America B 20, 2534 (2003).

180 D. Qu and D. Grischkowsky, “Observation of a new type of $\mathrm{THz}$ resonance of surface plasmons propagating on metal-film hole arrays," Physical Review Letters 93 (2004), 10.1103/physrevlett.93.196804.

181 J. H. Kang, D. S. Kim, and Q.-H. Park, "Local capacitor model for plasmonic electric field enhancement," Physical Review Letters 102 (2009), 10.1103/physrevlett.102.093906.
182 R. Gordon and A. G. Brolo, "Increased cut-off wavelength for a subwavelength hole in a real metal," Optics Express 13, 1933 (2005).

183 J. Bravo-Abad, A. I. Fernández-Domínguez, F. J. GarcíaVidal, and L. Martín-Moreno, "Theory of extraordinary transmission of light through quasiperiodic arrays of subwavelength holes," Physical Review Letters 99 (2007), 10.1103/physrevlett.99.203905.

184 Y. Takakura, "Optical resonance in a narrow slit in a thick metallic screen," Physical Review Letters 86, 5601 (2001).

185 V. Delgado and R. Marqués, "Surface impedance model for extraordinary transmission in 1d metallic and dielectric screens," Optics Express 19, 25290 (2011).

186 V. Galindo and C. Wu, "Numerical solutions for an infinite phased array of rectangular waveguides with thick walls," IEEE Transactions on Antennas and Propagation 14, 149 (1966).

187 P. Sheng, R. S. Stepleman, and P. N. Sanda, “Exact eigenfunctions for square-wave gratings: Application to diffraction and surface-plasmon calculations," Physical Review B 26, 2907 (1982).

188 F. J. Garcia-Vidal, L. Martin-Moreno, T. W. Ebbesen, and L. Kuipers, "Light passing through subwavelength apertures," Reviews of Modern Physics 82, 729 (2010).

189 H. Liu and P. Lalanne, "Microscopic theory of the extraordinary optical transmission," Nature 452, 728 (2008).

190 X.-Y. He, "Numerical analysis of the propagation properties of subwavelength semiconductor slit in the terahertz region," Optics Express 17, 15359 (2009).

191 P. Bell, J. Pendry, L. Moreno, and A. Ward, “A program for calculating photonic band structures and transmission coefficients of complex structures," Computer Physics Communications 85, 306 (1995).

192 L. Li, "New formulation of the fourier modal method for crossed surface-relief gratings," Journal of the Optical Society of America A 14, 2758 (1997).

193 L. Salomon, F. Grillot, A. V. Zayats, and F. de Fornel, "Near-field distribution of optical transmission of periodic subwavelength holes in a metal film," Physical Review Letters 86, 1110 (2001).

194 J. Zuloaga, E. Prodan, and P. Nordlander, “Quantum description of the plasmon resonances of a nanoparticle dimer," Nano Letters 9, 887 (2009).

195 R. Esteban, A. G. Borisov, P. Nordlander, and J. Aizpurua, "Bridging quantum and classical plasmonics with a quantum-corrected model," Nature Communications 3 (2012), 10.1038/ncomms1806.

196 D. Marinica, A. Kazansky, P. Nordlander, J. Aizpurua, and A. G. Borisov, "Quantum plasmonics: Nonlinear effects in the field enhancement of a plasmonic nanoparticle dimer," Nano Letters 12, 1333 (2012).

197 C. Ciraci, R. T. Hill, J. J. Mock, Y. Urzhumov, A. I. Fernandez-Dominguez, S. A. Maier, J. B. Pendry, A. Chilkoti, and D. R. Smith, "Probing the ultimate limits of plasmonic enhancement," Science 337, 1072 (2012).

198 M. S. Tame, K. R. McEnery, Ş. K. zdemir, J. Lee, S. A. Maier, and M. S. Kim, "Quantum plasmonics," Nature Physics 9, 329 (2013).

199 S. F. Tan, L. Wu, J. K. W. Yang, P. Bai, M. Bosman, and C. A. Nijhuis, "Quantum plasmon resonances controlled by molecular tunnel junctions," Science 343, 1496 (2014).

200 K. J. Savage, M. M. Hawkeye, R. Esteban, A. G. Borisov, J. Aizpurua, and J. J. Baumberg, "Revealing the quantum 
regime in tunnelling plasmonics," Nature 491, 574 (2012).

201 J. A. Scholl, A. García-Etxarri, A. L. Koh, and J. A. Dionne, "Observation of quantum tunneling between two plasmonic nanoparticles," Nano Letters 13, 564 (2013).

202 W. Zhu and K. B. Crozier, "Quantum mechanical limit to plasmonic enhancement as observed by surface-enhanced raman scattering," Nature Communications 5 (2014), $10.1038 /$ ncomms6228.

203 W. Zhu, R. Esteban, A. G. Borisov, J. J. Baumberg, P. Nordlander, H. J. Lezec, J. Aizpurua, and K. B. Crozier, "Quantum mechanical effects in plasmonic structures with subnanometre gaps," Nature Communications 7 (2016), $10.1038 /$ ncomms11495.

204 B. de Nijs, F. Benz, S. J. Barrow, D. O. Sigle, R. Chikkaraddy, A. Palma, C. Carnegie, M. Kamp, R. Sundararaman, P. Narang, O. A. Scherman, and J. J. Baumberg, "Plasmonic tunnel junctions for single-molecule redox chemistry," Nature Communications 8 (2017), 10.1038/s41467017-00819-7.

205 P. Cristofolini, G. Christmann, S. I. Tsintzos, G. Deligeorgis, G. Konstantinidis, Z. Hatzopoulos, P. G. Savvidis, and J. J. Baumberg, “Coupling quantum tunneling with cavity photons," Science 336, 704 (2012).

206 J. D. Mar, X. L. Xu, J. J. Baumberg, A. C. Irvine, C. Stanley, and D. A. Williams, "Voltage-controlled electron tunneling from a single self-assembled quantum dot embedded in a two-dimensional-electron-gas-based photovoltaic cell," Journal of Applied Physics 110, 053110 (2011).

207 N. Cazier, M. Buret, A. V. Uskov, L. Markey, J. Arocas, G. C. D. Francs, and A. Bouhelier, "Electrical excitation of waveguided surface plasmons by a light-emitting tunneling optical gap antenna," Optics Express 24, 3873 (2016).

208 A. V. Uskov, J. B. Khurgin, I. E. Protsenko, I. V. Smetanin, and A. Bouhelier, "Excitation of plasmonic nanoantennas by nonresonant and resonant electron tunnelling," Nanoscale 8, 14573 (2016).

209 A. Stolz, J. Berthelot, M.-M. Mennemanteuil, G. C. des Francs, L. Markey, V. Meunier, and A. Bouhelier, "Nonlinear photon-assisted tunneling transport in optical gap antennas," Nano Letters 14, 2330 (2014).

210 V. Kravtsov, S. Berweger, J. M. Atkin, and M. B. Raschke, "Control of plasmon emission and dynamics at the transition from classical to quantum coupling," Nano Letters 14, 5270 (2014).

211 S. C. H. Allen Taflove, Computational Electrodynamics: The Finite-Difference Time-Domain Method (ARTECH HOUSE INC, 2005).

212 J. D. Jackson, Classical electrodynamics, Third Edition (John Wiley \& Sons, 1998).

213 D. J. Griffiths, Introduction to electrodynamics; 4th ed. (Pearson, Boston, MA, 2013) re-published by Cambridge University Press in 2017.

214 C. Fattinger and D. Grischkowsky, "Point source terahertz optics," Applied Physics Letters 53, 1480 (1988).

215 C. Fattinger and D. Grischkowsky, "Terahertz beams," Applied Physics Letters 54, 490 (1989).

216 D. Grischkowsky, S. Keiding, M. van Exter, and C. Fattinger, "Far-infrared time-domain spectroscopy with terahertz beams of dielectrics and semiconductors," Journal of the Optical Society of America B 7, 2006 (1990).

217 S. J. Park, B. H. Son, S. J. Choi, H. S. Kim, and Y. H. Ahn, "Sensitive detection of yeast using terahertz slot an- tennas," Optics Express 22, 30467 (2014).

218 O. Sushko, R. Dubrovka, and R. S. Donnan, "Terahertz spectral domain computational analysis of hydration shell of proteins with increasingly complex tertiary structure," The Journal of Physical Chemistry B 117, 16486 (2013).

219 V. C. Nibali and M. Havenith, "New insights into the role of water in biological function: Studying solvated biomolecules using terahertz absorption spectroscopy in conjunction with molecular dynamics simulations," Journal of the American Chemical Society 136, 12800 (2014).

220 C. Rocchi, A. R. Bizzarri, and S. Cannistraro, "Water dynamical anomalies evidenced by molecular-dynamics simulations at the solvent-protein interface," Physical Review E 57, 3315 (1998).

221 M. Marchi, F. Sterpone, and M. Ceccarelli, "Water rotational relaxation and diffusion in hydrated lysozyme," Journal of the American Chemical Society 124, 6787 (2002).

222 N. Sengupta, S. Jaud, and D. J. Tobias, "Hydration dynamics in a partially denatured ensemble of the globular protein human $\alpha$-lactalbumin investigated with molecular dynamics simulations," Biophysical Journal 95, 5257 (2008).

223 S. K. Sinha, S. Chakraborty, and S. Bandyopadhyay, "Thickness of the hydration layer of a protein from molecular dynamics simulation," The Journal of Physical Chemistry B 112, 8203 (2008).

224 Y. Xu, R. Gnanasekaran, and D. M. Leitner, “Analysis of water and hydrogen bond dynamics at the surface of an antifreeze protein," Journal of Atomic, Molecular, and Optical Physics 2012, 1 (2012).

225 S. Chakraborty, S. K. Sinha, and S. Bandyopadhyay, "Lowfrequency vibrational spectrum of water in the hydration layer of a protein: a molecular dynamics simulation study," The Journal of Physical Chemistry B 111, 13626 (2007).

226 S. K. Sinha and S. Bandyopadhyay, "Differential flexibility of the secondary structures of lysozyme and the structure and ordering of surrounding water molecules," The Journal of Chemical Physics 134, 115101 (2011).

227 S. Bandyopadhyay, S. Chakraborty, and B. Bagchi, “Exploration of the secondary structure specific differential solvation dynamics between the native and molten globule states of the protein HP-36," The Journal of Physical Chemistry B 110, 20629 (2006).

228 S. Bandyopadhyay, S. Chakraborty, S. Balasubramanian, S. Pal, and B. Bagchi, "Atomistic simulation study of the coupled motion of amino acid residues and water molecules around protein HP-36: Fluctuations at and around the active sites," The Journal of Physical Chemistry B 108, 12608 (2004).

229 S. Pal and S. Bandyopadhyay, "Effects of protein conformational flexibilities and electrostatic interactions on the low-frequency vibrational spectrum of hydration water," The Journal of Physical Chemistry B 117, 5848 (2013).

230 S. Pal and S. Bandyopadhyay, "Importance of protein conformational motions and electrostatic anchoring sites on the dynamics and hydrogen bond properties of hydration water," Langmuir 29, 1162 (2013).

231 S. K. Sinha and S. Bandyopadhyay, "Polar solvation dynamics of lysozyme from molecular dynamics studies," The Journal of Chemical Physics 136, 185102 (2012).

232 S. K. Sinha and S. Bandyopadhyay, "Local heterogeneous dynamics of water around lysozyme: a computer simula- 
tion study," Phys. Chem. Chem. Phys. 14, 899 (2012).

233 T. Ding, T. Huber, A. P. Middelberg, and R. J. Falconer, "Characterization of low-frequency modes in aqueous peptides using far-infrared spectroscopy and molecular dynamics simulation," The Journal of Physical Chemistry A 115, 11559 (2011).

234 M. Heyden and M. Havenith, "Combining THz spectroscopy and MD simulations to study protein-hydration coupling," Methods 52, 74 (2010).

235 M. Heyden, J. Sun, H. Forbert, G. Mathias, M. Havenith, and D. Marx, "Understanding the origins of dipolar couplings and correlated motion in the vibrational spectrum of water," The Journal of Physical Chemistry Letters 3, 2135 (2012).

236 M. P. Allen et al., "Introduction to molecular dynamics simulation," Computational soft matter: from synthetic polymers to proteins 23, 1 (2004).

237 J. E. Bertie and Z. Lan, "Infrared intensities of liquids XX: The intensity of the $\mathrm{OH}$ stretching band of liquid water revisited, and the best current values of the optical constants of h2o(l) at $25^{\circ} \mathrm{C}$ between 15,000 and $1 \mathrm{~cm}-1,{ }^{\prime \prime}$ Applied Spectroscopy 50, 1047 (1996).

238 M. Heyden, J. Sun, S. Funkner, G. Mathias, H. Forbert, M. Havenith, and D. Marx, "Dissecting the THz spectrum of liquid water from first principles via correlations in time and space," Proceedings of the National Academy of Sciences 107, 12068 (2010).

239 J. Jiang, M. Xiao, S. Wang, K. Liu, X. Wang, and T. Liu, "Polarized low-coherence interferometer based on a matrix CCD and birefringence crystal with a two-dimensional angle," Optics Express 25, 15977 (2017).
240 H. liang Zhao, G. jun Ren, F. Liu, H. peng Xin, Y. bo Bai, and J. quan Yao, "Tunable terahertz source via liquid crystal grating coated with electron beam excited graphene: A theoretical analysis," Optics Communications 390, 137 (2017).

241 X. Gu, I.-T. Lin, and J.-M. Liu, "Extremely confined terahertz surface plasmon-polaritons in graphene-metal structures," Applied Physics Letters 103, 071103 (2013).

242 H. Zhao, Q. Guo, F. Xia, and H. Wang, “Two-dimensional materials for nanophotonics application," Nanophotonics 4 (2015), 10.1515/nanoph-2014-0022.

243 J. Conde, C. Bao, D. Cui, P. V. Baptista, and F. Tian, “Antibody-drug gold nanoantennas with raman spectroscopic fingerprints for in vivo tumour theranostics," Journal of Controlled Release 183, 87 (2014).

244 X. Huang and M. A. El-Sayed, “Gold nanoparticles: Optical properties and implementations in cancer diagnosis and photothermal therapy," Journal of Advanced Research 1, 13 (2010).

245 A. Abbas, M. J. Linman, and Q. Cheng, "New trends in instrumental design for surface plasmon resonance-based biosensors," Biosensors and Bioelectronics 26, 1815 (2011).

246 Y. Yanase, T. Hiragun, K. Ishii, T. Kawaguchi, T. Yanase, M. Kawai, K. Sakamoto, and M. Hide, "Surface plasmon resonance for cell-based clinical diagnosis," Sensors 14, 4948 (2014).

247 E. Helmerhorst, D. J. Chandler, M. Nussio, and C. D. Mamotte, "Real-time and label-free bio-sensing of molecular interactions by surface plasmon resonance: A laboratory medicine perspective," The Clinical biochemist. Reviews 33, 161 (2012).

248 S. L. Dodson, C. Cao, H. Zaribafzadeh, S. Li, and Q. Xiong, "Engineering plasmonic nanorod arrays for colon cancer marker detection," Biosensors and Bioelectronics 63, 472 (2015). 